\title{
Chapitre 2 \\ Conception et fonctionnement d'un réacteur à eau sous pression
}

\subsection{Généralités sur le fonctionnement d'un réacteur}

Les noyaux de certains isotopes contenus dans le combustible nucléaire, tels que $\mathrm{l}^{\prime 235} \mathrm{U}$ et le ${ }^{239} \mathrm{Pu}$, peuvent être scindés par fission en deux fragments plus petits appelés produits de fission. Ces fragments ont une grande énergie cinétique qui se dégage principalement sous forme d'énergie cinétique d'agitation thermique dans le matériau combustible environnant. Ce dégagement d'énergie est utilisé dans les réacteurs de puissance pour produire de l'électricité. La réaction de fission en deux fragments peut se produire soit sous l'action de neutrons (on parle de « fission induite »), soit spontanément pour les isotopes de masse élevée (on parle alors de « fission spontanée »). La fission est accompagnée de la production de deux à trois neutrons par fission dont certains peuvent soit conduire, à leur tour, à d'autres fissions - c'est le principe de la réaction en chaîne - soit être absorbés dans le combustible sans induire de fission nucléaire, soit s'en échapper.

Les neutrons produits par fission à partir des neutrons d'une génération constituent une nouvelle génération de neutrons. Le facteur d'efficacité multiplicatif des neutrons, $k$, est le nombre moyen de neutrons émis par les fissions qui vont engendrer une nouvelle génération. La valeur de $k$ gouverne l'évolution de la réaction en chaîne :

- si $k<1$, le système est dit « sous-critique », le système ne peut pas entretenir une réaction en chaîne et elle finit par s'éteindre ; 
- si $k=1$, le système est dit « critique », c'est-à-dire qu'il apparaît autant de neutrons qu'il en disparaît, la réaction est exactement entretenue. Cette situation conduit à un niveau de puissance produite constant ;

- si $k>1$, le système est dit «sur critique "; pour chaque fission, il y aura en moyenne $k$ fissions à la génération suivante; le nombre de fissions croît donc exponentiellement.

On distingue en fait deux types de situation "sur critique »: la sur criticité prompte et la sur criticité retardée. La quasi-totalité des neutrons de fission sont émis instantanément (par exemple, $99,3 \%$ des neutrons sont émis en $10^{-7} \mathrm{~s}$ pour $\left.\mathrm{l}^{235} \mathrm{U}\right)$; ces neutrons sont appelés neutrons prompts. Cependant, pour une faible fraction des produits de fission, il y a d'abord une désexcitation du produit de fission par radioactivité $\beta$, avant l'émission de neutrons dits « retardés ». Ces neutrons sont émis selon la période de la désintégration $\beta$, soit en quelques dixièmes à quelques dizaines de secondes. La fraction de neutrons retardés est typiquement inférieure à un pour cent du nombre total de neutrons générés à un instant donné dans une réaction en chaîne. Dans l'intervalle compris entre $k=1$ et $k=1 /(1-\beta) \approx 1+\beta$, la sur criticité est dite retardée ; pour $k>1 / 1(1-\beta) \approx 1+\beta$, la sur criticité est dite prompte. La valeur de la fraction de neutrons retardés qui représente l'écart entre sur criticité retardée et sur criticité prompte est également appelée « dollar » et elle dépend de l'isotope considéré.

Pour la production d'énergie, les réacteurs nucléaires opèrent dans la zone de sur criticité retardée, car, dans cette zone, grâce à la présence des neutrons retardés, les évolutions de puissance se font beaucoup plus lentement qu'avec les neutrons prompts uniquement. Sans les neutrons retardés, les changements de puissance d'un réacteur seraient beaucoup trop rapides pour être maîtrisés par des systèmes d'absorption des neutrons.

Une autre grandeur est couramment utilisée pour traduire l'écart du système par rapport à l'état critique; il s'agit de la réactivité $\rho, \rho=1-1 / k$. Les valeurs de $\rho$ positives correspondent à des états "sur critiques » et les valeurs négatives à des états « sous-critiques ».

Dans un réacteur nucléaire, la réaction en chaîne doit être maîtrisée, c'est-à-dire qu'une valeur de réactivité nulle ou négative doit être maintenue par l'utilisation d'éléments absorbant les neutrons, situés pour les réacteurs à eau sous pression dans des organes mobiles appelés barres de commande (cadmium ou bore par exemple) ou dissous dans l'eau de refroidissement (bore).

Dans certaines situations accidentelles de faible probabilité, la réactivité du réacteur peut atteindre des valeurs positives élevées de sorte que la réaction en chaîne devient sur critique. Ces situations pourraient conduire, si les dispositions prises pour ramener le réacteur dans un domaine de fonctionnement sûr étaient insuffisantes, à une augmentation de la puissance non maîtrisable par les barres de commande. Une telle situation pourrait conduire à un endommagement sévère du réacteur ; c'est notamment ce qui s'est produit lors de l'accident de Tchernobyl (§ 7.2). 
Dans un réacteur, la réactivité varie principalement en fonction de la température du combustible, de celle du fluide réfrigérant et du taux de vide du réfrigérant. L'influence de chacun de ces paramètres est caractérisée par un coefficient de réactivité qui est la dérivée de la réactivité par rapport au paramètre considéré. Pour le combustible, une augmentation de puissance se traduit par une augmentation de la température du combustible et un accroissement de la capture des neutrons par $l^{238} \mathrm{U}$; le coefficient de réactivité, appelé coefficient de température ou coefficient Doppler, est par conséquent négatif. Pour le fluide réfrigérant, le coefficient de réactivité est lié à la variation de la densité du fluide (coefficient de température) ou du taux de vide (coefficient de vide). Ces coefficients sont négatifs dans le cas des réacteurs à eau sous pression ${ }^{1}$ de façon à assurer la stabilité du réacteur et à limiter la puissance maximale susceptible d'être atteinte en situation accidentelle.

Certains des produits de fission formés sont radioactifs. Cette radioactivité se traduit, même après l'arrêt de la réaction en chaîne, par une libération d'énergie sous forme de chaleur, appelée "puissance résiduelle », qui décroît en fonction du temps et qui représente, une heure après l'arrêt du réacteur, environ 1,5\% de sa puissance en fonctionnement ${ }^{2}$.

L'énergie libérée par les fissions et par les produits de fission doit être évacuée en permanence pour éviter une montée en température excessive du réacteur. Dans les réacteurs à eau sous pression, lors du fonctionnement normal en puissance, l'évacuation de l'énergie est réalisée grâce à trois circuits successifs, en vue notamment d'éviter le rejet d'eau radioactive provenant du cœur à l'extérieur de la centrale (figure 2.3) :

- le circuit primaire qui permet de refroidir le cœur par une circulation d'eau à une température moyenne d'environ $300^{\circ} \mathrm{C}$ et à une pression de 155 bars ;

- le circuit secondaire qui évacue l'énergie du circuit primaire par l'intermédiaire de générateurs de vapeur qui alimentent en vapeur le groupe turboalternateur produisant l'électricité ;

- le circuit tertiaire qui comporte un condenseur et évacue l'énergie restante vers une rivière, un fleuve ou la mer ou dans l'atmosphère par des tours de réfrigération.

Cette brève description du fonctionnement d'un réacteur nucléaire identifie les fonctions fondamentales de sûreté qui doivent être assurées en permanence :

- la maîtrise de la réactivité ;

- l'évacuation de la puissance ;

1. Dans les réacteurs à eau sous pression, l'eau joue le rôle de « modérateur ». L'eau permet en effet de ralentir les neutrons produits par les fissions (ceux-ci cèdent leur énergie cinétique par chocs avec les noyaux des atomes d'hydrogène de l'eau) et d'accroître le rendement des réactions de fission. Lorsque la température dans le cœur du réacteur augmente, la densité de l'eau diminue si bien que le ralentissement des neutrons devient moins efficace ce qui a pour effet de réduire les réactions de fission. Le coefficient de température de l'eau est donc négatif.

2. Une heure après l'arrêt du réacteur, la puissance thermique dégagée est de $40 \mathrm{MW}$ pour un réacteur de $900 \mathrm{MWe}$ et de $58 \mathrm{MW}$ pour un réacteur de $1300 \mathrm{MWe}$; un jour après l'arrêt, elle est de $16 \mathrm{MW}$ pour un réacteur de $900 \mathrm{MWe}$ et de $24 \mathrm{MW}$ pour un réacteur de $1300 \mathrm{MWe}$. 
- le confinement des produits de fission et plus généralement de la radioactivité (certains produits d'activation présents dans le circuit primaire ${ }^{3}$ sont également radioactifs).

\subsection{Les réacteurs à eau sous pression du parc électronucléaire français}

Différents types de réacteurs nucléaires sont utilisés pour la production d'électricité. Ils utilisent différentes matières fissiles (uranium naturel, uranium enrichi en isotope 235 , plutonium, etc.) et différents modérateurs de la réaction nucléaire (graphite, eau, eau lourde, etc. $)^{4}$. Ils sont également caractérisés par la nature du fluide (eau ordinaire sous forme de liquide ou de vapeur, eau lourde, gaz, sodium, etc.) servant à évacuer la chaleur du cœur où ont lieu les réactions de fission pour la transmettre aux circuits alimentant les turboalternateurs ou directement aux turboalternateurs eux-mêmes.

Les réacteurs électrogènes actuellement en service en France utilisent de l'uranium enrichi sous forme d'oxyde, éventuellement mélangé à de l'oxyde de plutonium provenant du traitement de combustibles usés, et, comme fluide de transfert de la chaleur, de l'eau ordinaire sous une pression suffisamment élevée pour rester liquide (155 bars) à sa température de fonctionnement $\left(300^{\circ} \mathrm{C}\right)$. Ils sont appelés « réacteurs à eau sous pression » (REP) et appartiennent à ce que l'on dénomme communément la deuxième génération de réacteurs nucléaires de puissance ${ }^{5}$.

Une particularité française est la standardisation du parc, avec un nombre important de réacteurs techniquement proches, qui justifie une présentation générique de ceuxci dans le présent chapitre. Les 19 centrales nucléaires en exploitation en France comportent de deux à six réacteurs à eau sous pression, pour un total de 58 réacteurs. Ce parc de réacteurs nucléaires est constitué par des séries de réacteurs appelées paliers: $900 \mathrm{MWe}, 1300 \mathrm{MWe}, 1450 \mathrm{MWe}$ (ou N4) (figure 2.1).

Les 34 réacteurs de 900 MWe sont répartis en deux principaux types:

- le CPO, constitué des deux réacteurs de Fessenheim et des quatre réacteurs du Bugey ;

- le CPY (constitué des types CP1 et CP2) qui regroupe les 28 autres réacteurs (quatre réacteurs au Blayais, quatre à Dampierre, six à Gravelines, quatre au Tricastin, quatre à Chinon, quatre à Cruas-Meysse et deux à Saint-Laurent des Eaux).

3. Des produits radioactifs peuvent être formés sous rayonnement par activation des composants métalliques du circuit primaire puis être transférés dans le fluide primaire sous l'effet de mécanismes de corrosion.

4. Le « modérateur » ralentit la vitesse des neutrons et augmente ainsi la probabilité qu'ils provoquent une fission.

5. La première génération regroupe les réacteurs construits avant les années 1970. En France, les réacteurs de première génération étaient des réacteurs de type uranium naturel-graphite-gaz (filière UNGG). 
Les 20 réacteurs de $1300 \mathrm{MWe}$ sont répartis en deux principaux types :

- le P4, constitué de huit réacteurs dont deux à Flamanville, quatre à Paluel et deux à Saint-Alban ;

- le $\mathrm{P}^{\prime} 4$, constitué de douze réacteurs dont deux à Belleville-sur-Loire, quatre à Cattenom, deux à Golfech, deux à Nogent-sur-Seine et deux à Penly.

Enfin, le palier N4 est constitué de quatre réacteurs de $1450 \mathrm{MWe}$, dont deux sur le site de Chooz et deux sur le site de Civaux.

Malgré la standardisation voulue du parc de réacteurs électronucléaires français, des nouveautés technologiques ont été introduites au fur et à mesure de la conception et de la réalisation des centrales. La construction du parc s'est faite en quatre étapes principales :

- le 900 MWe, « présérie » de type CPO, mis en exploitation entre 1977 et 1979 ;

- le 900 MWe de type CPY, mis en exploitation entre 1980 et 1987 ;

- le 1300 MWe de types P4 et P'4, mis en exploitation entre 1984 et 1993 ;

- le 1450 MWe (ou N4) mis en exploitation entre 2000 et 2002.

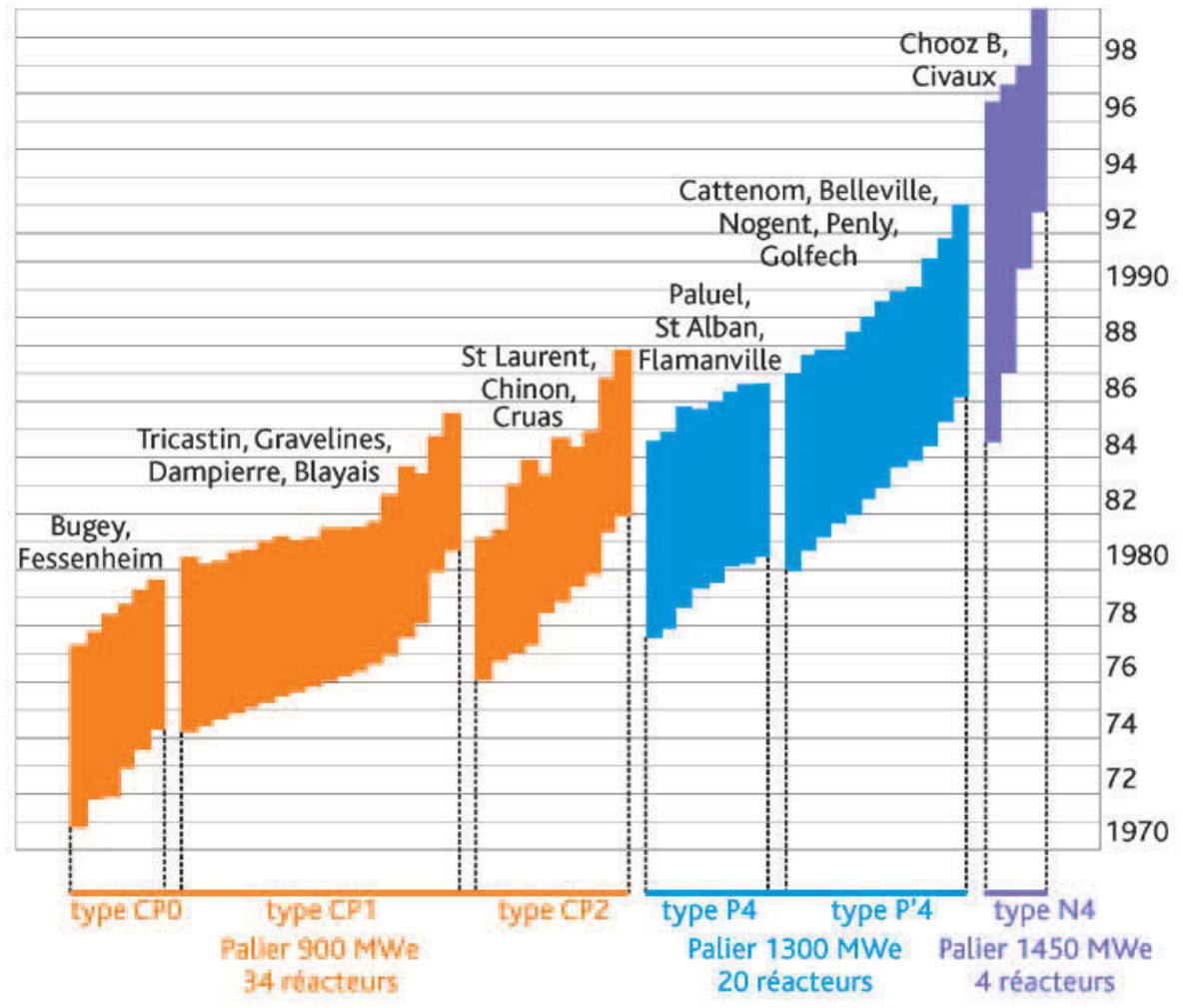

Figure 2.1. Années de construction et répartition des trois paliers de réacteurs de puissance 900, 1300 et 1450 MWe en exploitation en France en 2013. 
Les réacteurs de type CPY ont bénéficié du retour d'expérience des études de conception, de la construction et de l'exploitation de ceux du type CPO. Contrairement aux études de conception du type CPO qui ont été conduites séparément pour chacun des deux sites, les études de conception du type CPY ont été réalisées pour l'ensemble des sites d'implantation. Le type CPY diffère ainsi du type CPO par la conception des bâtiments, et notamment par celle de l'enceinte de confinement, modifiée pour y faciliter les opérations d'exploitation, par l'implantation des systèmes de sauvegarde, modifiée pour accroître l'indépendance des voies de ces systèmes et améliorer leur fiabilité, et par un pilotage plus souple du réacteur (notamment par l'utilisation de grappes de commande et l'ajout de grappes de commande moins absorbantes pour les neutrons ${ }^{6}$ ). Pour les réacteurs de type CP2 l'orientation de la salle des machines a été changée de $90^{\circ}$ afin d'éviter que des projectiles résultant $d^{\prime}$ une rupture du turboalternateur puissent endommager l'enceinte de confinement du réacteur (figure 2.2).

Les réacteurs de 1300 MWe différent des réacteurs de 900 MWe par la conception du cœur, des circuits et du système de protection du cœur du réacteur ainsi que des bâtiments de l'installation. L'augmentation de puissance a été obtenue par un accroissement de la taille du cœur. La capacité de refroidissement du circuit primaire a été accrue pour pouvoir évacuer l'augmentation de puissance (de 900 à $1300 \mathrm{MWe}$ ) grâce à la mise en place d'une boucle de refroidissement supplémentaire (le nombre de boucles passe ainsi de trois pour les réacteurs de $900 \mathrm{MWe}$ à quatre pour les réacteurs de $1300 \mathrm{MWe}$ ) (figure 2.3). Les composants de chaque boucle primaire ont également une taille plus importante par rapport au palier précédent. En termes d'implantation des bâtiments, les tranches sont indépendantes alors qu'elles étaient jumelées pour les paliers précédents (figure 2.2). Les systèmes de sauvegarde et les systèmes auxiliaires sont implantés dans des bâtiments spécifiques à chaque tranche pour améliorer la sûreté de leur fonctionnement. En outre, chaque enceinte de confinement comporte une double paroi en béton (paroi interne en béton précontraint et paroi externe en béton armé) remplaçant la seule paroi en béton précontraint doublée d'une peau d'étanchéité en acier des réacteurs de $900 \mathrm{MWe}$. De nouvelles technologies de contrôle-commande, à base de microprocesseurs et de mémoires programmables, sont mises en œuvre. Le type P'4 se démarque du type P4 par une recherche d'optimisation de l'installation des bâtiments et des ouvrages, dans le but essentiel de réduire les coûts ; ceci se traduit par un plan de masse plus compact et des bâtiments et des structures de dimensions plus réduites.

6. Les grappes de commande sont constituées de 24 crayons. Il existe deux types de grappes de commande, "noires» et "grises», le premier - grappe «noire »-comportant 24 crayons absorbant les neutrons (composées d'un alliage d'argent, d'indium et de cadmium (AIC) ou de carbure de bore $\left(\mathrm{B}_{4} \mathrm{C}\right)$ ), l'autre - grappe " grise »-comportant des crayons composés de matière plus ou moins absorbante (par exemple seulement huit crayons absorbant en $\mathrm{AIC}$ ou $\mathrm{B}_{4} \mathrm{C}$, les 18 autres crayons étant en acier, plus transparent aux neutrons). Les mouvements différenciés de ces grappes dans le cœur permettent d'optimiser la distribution spatiale de puissance, de piloter les variations de puissance du réacteur et de réguler la température moyenne de l'eau du circuit primaire. 
Enfin, les réacteurs de $1450 \mathrm{MWe}$ se distinguent notamment de ceux des paliers précédents par un accroissement de la taille du cœur du réacteur, une conception plus compacte des générateurs de vapeur (GV) délivrant de la vapeur à plus haute pression, par la conception du groupe motopompe primaire (accroissement du débit) et par l'informatisation de la conduite.

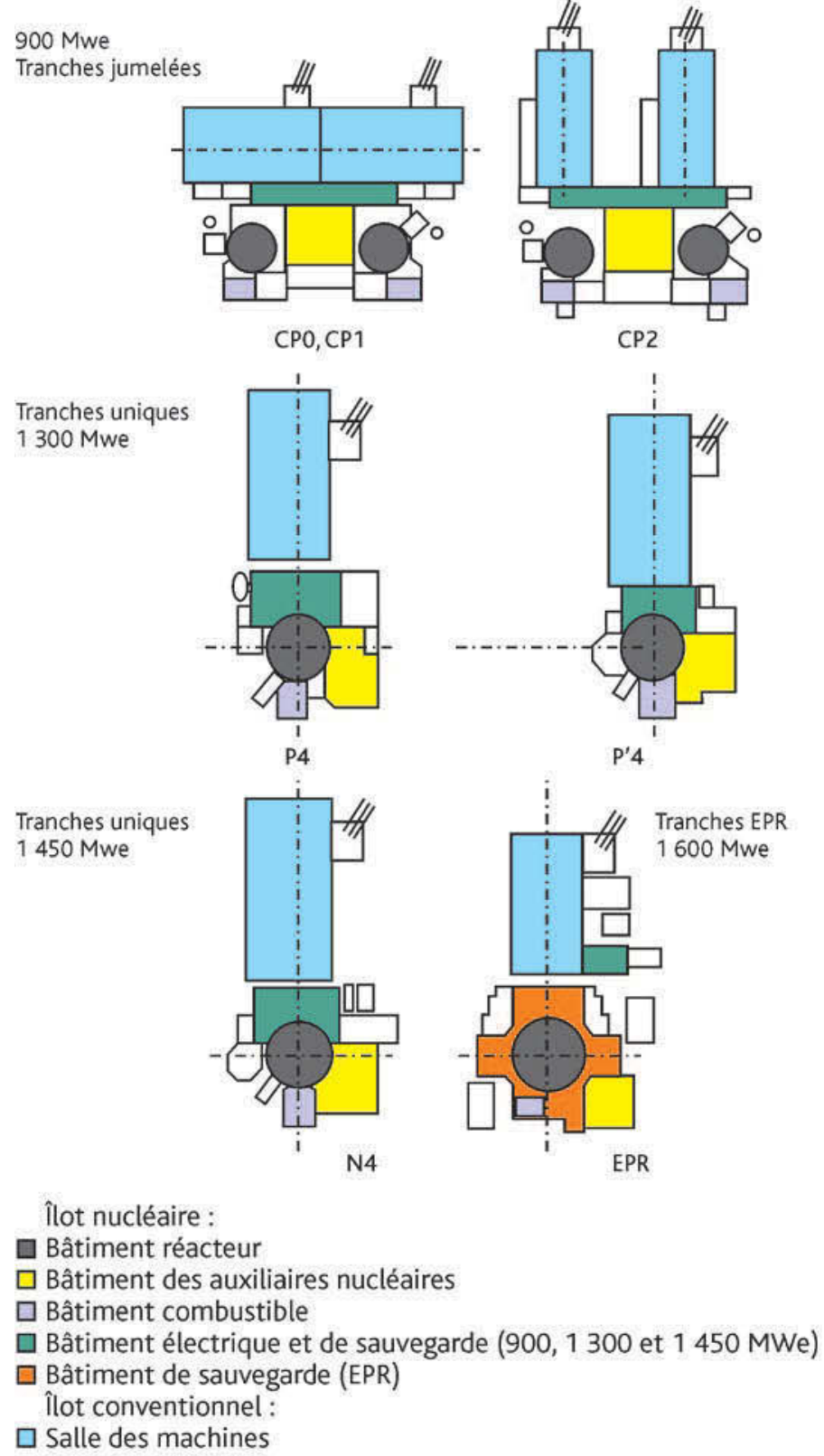

Figure 2.2. Plan de masse schématique des bâtiments des différents paliers de réacteurs en France. 
La nouvelle génération de réacteurs qu'EDF envisage d'exploiter en France sera constituée de réacteurs dits de troisième génération de type EPR (pour European Pressurized water Reactor). Un réacteur d'une puissance d'environ 1600 MWe est en construction sur le site de Flamanville dans la Manche. Ces nouveaux réacteurs à eau sous pression sont conçus de manière " évolutionnaire » par rapport aux réacteurs actuellement en exploitation en France ; ils bénéficient de ce fait du retour d'expérience important obtenu par l'exploitation du parc actuel et répondent à des objectifs de sûreté renforcés. Ils bénéficient également des résultats de la recherche, notamment dans le domaine des accidents de fusion du cœur qui ont été pris en compte dès la conception. Ils diffèrent notamment des REP de deuxième génération par la conception des circuits et du système de protection du cœur du réacteur et des bâtiments de l'installation (en particulier l'enceinte de confinement) qui offre une protection accrue en cas d'accident.

La conception du circuit primaire, la configuration des boucles et la conception des principaux composants sont très proches de celles du palier N4. Les évolutions notables sont :

- l'augmentation des volumes d'eau primaire et secondaire (notamment dans les générateurs de vapeur) afin d'accroître l'inertie thermique du réacteur ;

- l'organisation des systèmes de sauvegarde et des systèmes supports (injection de sécurité (RIS), alimentation de secours en eau des générateurs de vapeur (ASG), refroidissement intermédiaire (RRI) et eau brute secouru (SEC), alimentations électriques de secours (AES)) en quatre trains indépendants, placés dans des locaux séparés physiquement. La séparation physique des systèmes de sauvegarde vise à assurer leur disponibilité en cas d'agression interne ou externe (e.g., incendie, séisme, inondation).

Pour l'enceinte de confinement, outre le renforcement de la structure (plus particulièrement de la paroi externe en béton, voir $§$ 2.3.2.3), les modifications suivantes ont été apportées par rapport à celles du palier N4 :

- implantation à l'intérieur de l'enceinte de confinement du réservoir d'eau borée IRWST (initiales de l'appellation anglaise In-containment Refueling Water Storage Tank), alimentant l'injection de sécurité et les circuits de refroidissement de l'enceinte CHRS, (initiales de l'appellation anglaise Containment Heat Removal System) ;

- installation d'un dispositif pour récupérer et refroidir le corium dans un compartiment spécifique après la défaillance de la cuve lors d'un accident avec fusion du cœur ; ce dispositif vise à assurer l'étanchéité du radier de l'enceinte de confinement sur le long terme si un tel accident de fusion du cœur survenait ;

- mise en place d'une peau d'étanchéité sur la paroi interne de l'enceinte à double paroi.

Une autre différence notable par rapport au palier N4 est que les locaux protégés par la paroi en béton externe renforcée (coque avion) sont plus nombreux : en plus du bâtiment du réacteur, la paroi en béton externe couvre le bâtiment du combustible et deux des locaux contenant les systèmes de sauvegarde. 
Le plan de masse des bâtiments (figure 2.2) a notamment évolué pour permettre l'implantation dans des locaux séparés des quatre trains indépendants des systèmes de sauvegarde et des systèmes supports et pour éviter les fuites directes de l'enceinte de confinement vers l'environnement. Les traversées de l'enceinte débouchent toutes dans des bâtiments à la périphérie du bâtiment du réacteur, ventilés et équipés de moyens de filtration.

Dans la suite de ce chapitre, sont présentés de manière relativement générique et synthétique les principaux constituants des réacteurs en exploitation en France ainsi que le fonctionnement de ces réacteurs en situation normale et accidentelle, de manière à fournir les éléments de compréhension nécessaires à la lecture de l'ouvrage. Les spécificités du réacteur EPR sont décrites lorsqu'elles concernent la prise en compte des accidents de fusion du cœur.

\subsection{Description d'un réacteur à eau sous pression et de ses principaux circuits}

\subsubsection{Présentation générale de l'installation}

Chaque réacteur comprend un îlot nucléaire, un îlot conventionnel, des ouvrages de prise et de rejet d'eau et éventuellement un aéroréfrigérant (figures 2.2 et 2.3).

L'îlot nucléaire comporte principalement :

- le bâtiment du réacteur (BR) qui contient le réacteur et l'ensemble des circuits primaires sous pression ainsi qu'une partie des circuits et systèmes assurant le fonctionnement et la sûreté du réacteur (figures 2.3, 2.6 et 2.7) ;

- le bâtiment du combustible (BK) abritant les installations d'entreposage et de manutention du combustible neuf (en attente de chargement dans le réacteur) et irradié (en attente de transfert vers les usines de retraitement). Il comprend aussi les matériels du circuit de refroidissement et de traitement des eaux de piscine (PTR) et, pour les tranches en exploitation, les matériels du circuit de refroidissement de secours des générateurs de vapeur (ASG). Le réacteur EPR est quant à lui équipé de quatre trains indépendants d'alimentation de secours des générateurs de vapeur, chaque train étant implanté dans une des quatre divisions du bâtiment des auxiliaires de sauvegarde ;

- un bâtiment des auxiliaires de sauvegarde et des locaux électriques (BAS/BL) qui abrite en sa partie inférieure les principaux systèmes de sauvegarde et en sa partie supérieure les locaux électriques. Ces deux parties ne communiquent pas entre elles. Les locaux des auxiliaires de sauvegarde contiennent notamment les matériels du circuit d'injection de sécurité (RIS), du circuit d'aspersion dans l'enceinte (EAS), du circuit de refroidissement intermédiaire (RRI) ainsi que les matériels nécessaires à la ventilation. Les locaux électriques abritent l'ensemble des moyens de commande de la tranche (la salle de commande et les locaux d'exploitation, les alimentations électriques, le contrôle-commande de la tranche). Il est à noter que, pour les 900 MWe, il n'y a qu'un bâtiment des auxiliaires de sauvegarde et des locaux électriques pour deux tranches « jumelées »; pour les 


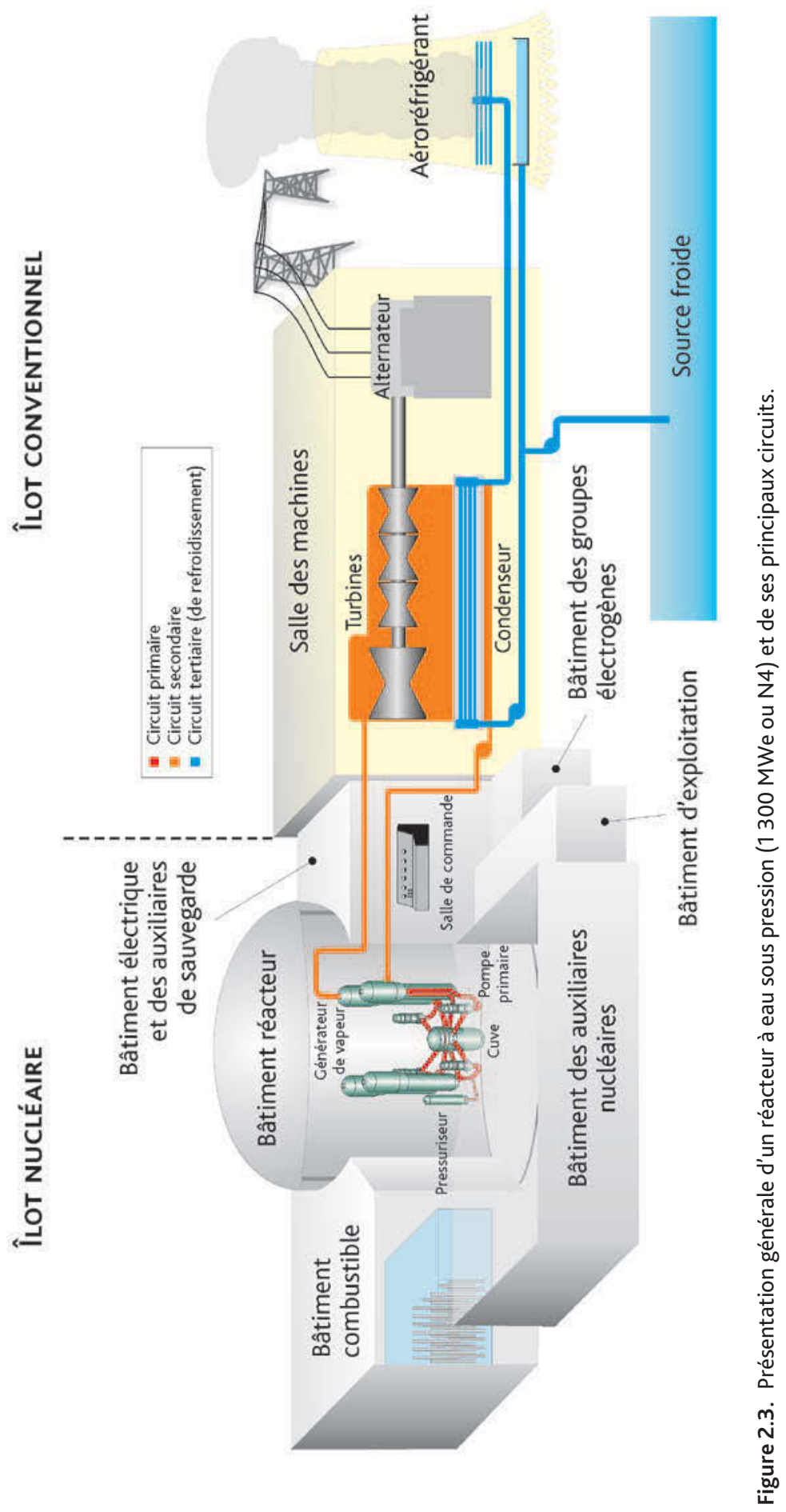


paliers 1300 MWe et N4, il y a un bâtiment par tranche; pour EPR, il y a quatre systèmes de sauvegarde indépendants implantés chacun avec ses systèmes supports dans un local séparé physiquement des autres, appelé « division » du bâtiment des auxiliaires de sauvegarde. Deux « divisions » du bâtiment des auxiliaires de sauvegarde sont protégées par la paroi externe en béton renforcée (il s'agit des « divisions » 2 et 3 ). La salle de contrôle commande se situe dans la « division » 3 du bâtiment des auxiliaires de sauvegarde ;

- un bâtiment des auxiliaires nucléaires (BAN) qui abrite les circuits auxiliaires nécessaires au fonctionnement normal du réacteur. Il abrite notamment les matériels des circuits de contrôle chimique et volumétrique (RCV), de traitement des effluents gazeux, de traitement des effluents primaires et d'acide borique ;

- deux bâtiments séparés géographiquement qui abritent chaucun un groupe électrogène à moteur diesel (alimentation électrique de sauvegarde). Pour EPR, les alimentations électriques externes de sauvegarde comprennent quatre groupes électrogènes à moteur diesel par bâtiment situés dans deux bâtiments dédiés et deux autres groupes électrogènes d'ultime secours ;

- un bâtiment d'exploitation.

Les équipements de l'îlot conventionnel fournissent l'énergie électrique au réseau de transport à partir de la vapeur produite dans l'îlot nucléaire. L'îlot conventionnel comporte notamment :

- la salle des machines qui abrite le groupe turboalternateur qui récupère la vapeur produite pour la transformer en électricité et ses auxiliaires ;

- une station de pompage qui permet d'assurer le refroidissement de l'installation en fonctionnement normal, ainsi que le refroidissement de secours avec les ouvrages d'eau connexes ;

- un aérofrigérant si la réfrigération de la tranche est réalisée en circuit fermé.

Certains de ces matériels participent à la sûreté du réacteur. En particulier, les circuits secondaires appartiennent pour partie à l'îlot nucléaire et pour partie à l'îlot conventionnel.

\subsubsection{Description des principaux constituants d'un REP}

\subsubsection{Le cœur du réacteur}

Le cœur du réacteur est composé d'assemblages combustibles (figure 2.4). Chaque assemblage comporte 264 crayons combustibles (figure 2.4, partie gauche), 24 tubes pouvant contenir les crayons d'une grappe de commande et un tube d'instrumentation, disposés suivant un réseau carré $17 \times 17$ (figure 2.4, partie droite). Les crayons combustibles sont constitués de tubes en alliage de zirconium appelés aussi gaines (le zirconium absorbe très faiblement les neutrons et résiste bien à la corrosion). L'alliage le plus fréquemment utilisé dans les REP français est le zircaloy comportant $98 \%$ de zirconium. Les gaines, de 0,6 mm d'épaisseur et de 9,5 mm de diamètre, sont maintenues par des grilles en zircaloy. À l'intérieur des crayons sont empilées des pastilles de $8,2 \mathrm{~mm}$ de diamètre de dioxyde d'uranium $\left(\mathrm{UO}_{2}\right)$ ou 

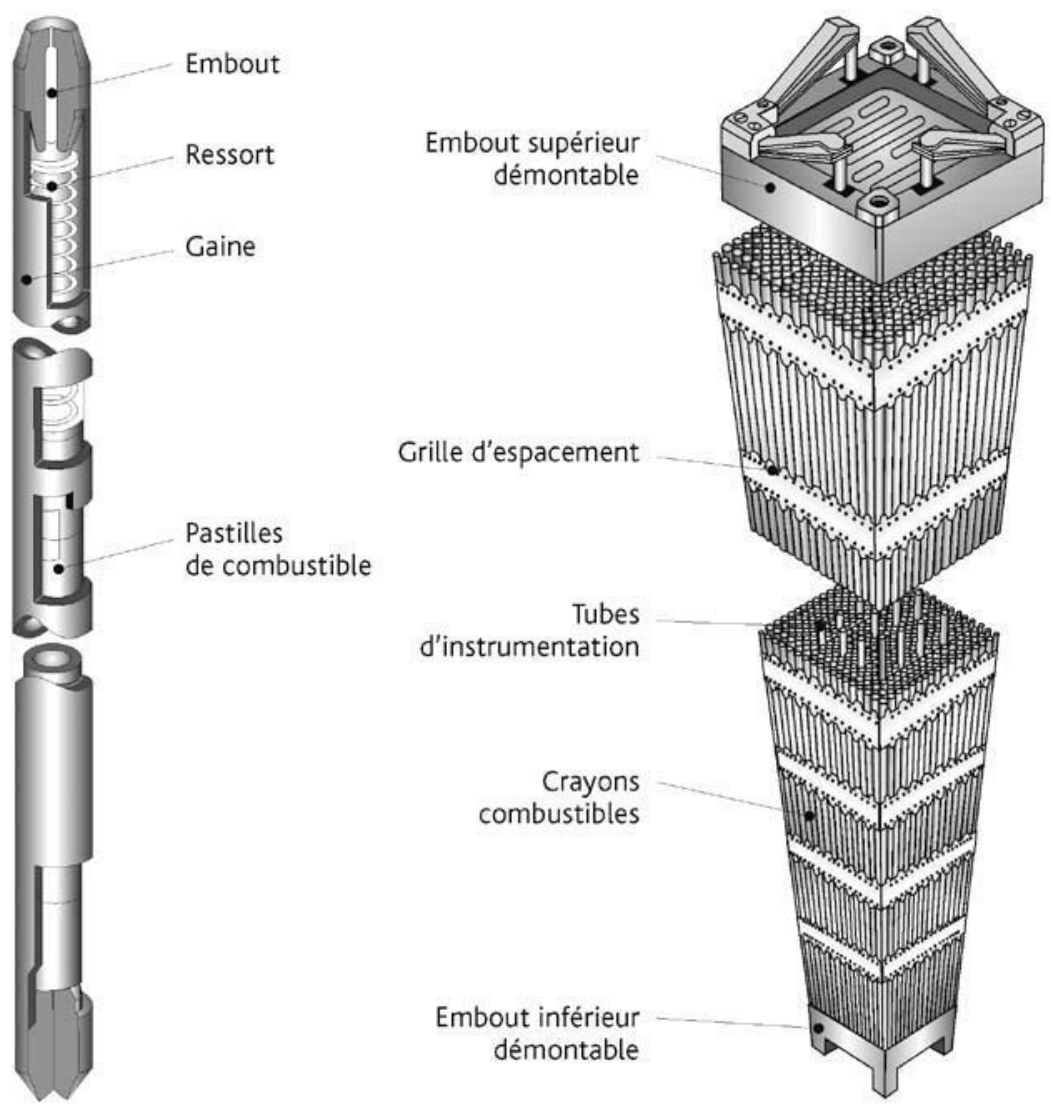

Figure 2.4. Schéma d'un crayon combustible (gauche) et des principaux constituants d'un assemblage combustible (droite).

d'un mélange d'oxydes d'uranium et de plutonium ((U,Pu) $\mathrm{O}_{2}$, combustible dit MOX), qui constituent le combustible nucléaire. L'enrichissement en ${ }^{235} \mathrm{U}$ varie de $3 \%$ à $4,5 \%$ suivant le mode de gestion du combustible. Les assemblages combustibles sont semblables pour tous les paliers, seule leur longueur varie. Le combustible est renouvelé lors des arrêts du réacteur d'une périodicité de 12 à 18 mois, par tiers ou quart de cœur.

Les principales caractéristiques du combustible et du cœur sont rappelées dans le tableau 2.1 pour les différents paliers.

7. Le combustible voit sa matière fissile diminuer au cours du fonctionnement du réacteur et les assemblages de crayons combustibles usés doivent être remplacés par des assemblages neufs. Il existe différents modes de gestion de ces remplacements, notamment en fonction de l'enrichissement initial en matière fissile du combustible. 
Tableau 2.1. Caractéristiques des cœurs des différents paliers.

\begin{tabular}{|l|c|c|c|c|}
\hline \multicolumn{1}{|c|}{ Palier } & $900 \mathrm{MWe}$ & $1300 \mathrm{MWe}$ & $1450 \mathrm{MWe}$ & EPR \\
\hline $\begin{array}{l}\text { Nombre d'assemblages com- } \\
\text { bustibles }\end{array}$ & 157 & 193 & 205 & 241 \\
\hline $\begin{array}{l}\text { Hauteur totale des pastilles } \\
\text { de combustible dans chaque } \\
\text { crayon de l'assemblage }(m)\end{array}$ & 3,66 & 4,27 & 4,27 & 4,20 \\
\hline $\begin{array}{l}\text { Nombre de grappes } \\
\text { de commande } \\
\text { Matériau absorbant }\end{array}$ & 57 & 65 & 73 & 89 \\
\hline Masse d'uranium enrichi $(t)$ & 72,5 & $\mathrm{Ag}-\operatorname{In}-\mathrm{Cd}+\mathrm{B}_{4} \mathrm{C}$ & $\mathrm{Ag}-\mathrm{In}-\mathrm{Cd}+\mathrm{B}_{4} \mathrm{C}$ & $\mathrm{Ag}-\ln -\mathrm{Cd}+\mathrm{B}_{4} \mathrm{C}$ \\
\hline
\end{tabular}

Le cœur est disposé à l'intérieur d'une cuve en acier au carbone 16MND5 munie d'un couvercle qui est enlevé pour les opérations de renouvellement du combustible (figure 2.5). À l'intérieur de la cuve sont disposées des structures métalliques qui peuvent être totalement retirées pour les inspections périodiques :

- les structures inférieures assurent le supportage du cœur ;

- les structures latérales (enveloppe du cœur) assurent la séparation entre le fluide froid pénétrant dans la cuve et le fluide chaud sortant du cœur ;

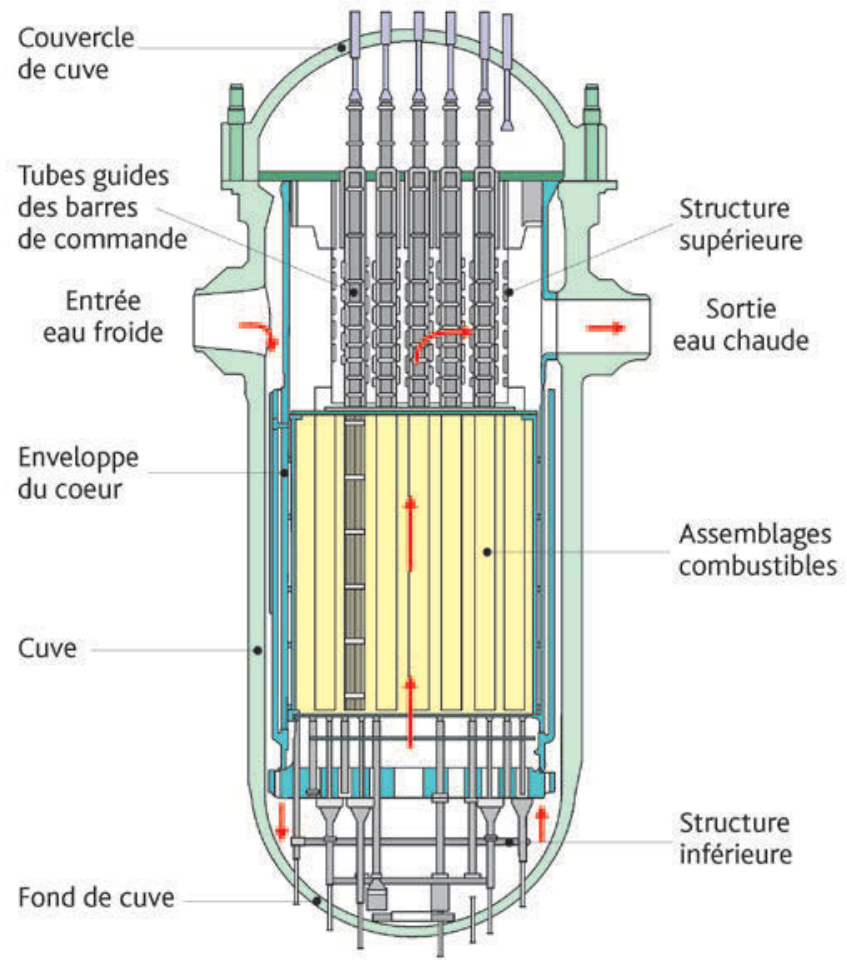

Figure 2.5. Schéma de la cuve d'un réacteur REP (exemple de Fessenheim). 
- les structures supérieures sont constituées par les tubes guides des barres de commande.

Les dimensions des cuves des différents paliers sont indiquées dans le tableau 2.2.

Tableau 2.2. Dimensions des cuves des différents paliers.

\begin{tabular}{|l|c|c|c|c|}
\hline \multicolumn{1}{|c|}{ Palier } & $900 \mathrm{MWe}$ & $1300 \mathrm{MWe}$ & $1450 \mathrm{MWe}$ & EPR \\
\hline Diamètre intérieur $(\mathrm{m})$ & 4,00 & 4,39 & 4,486 & 4,885 \\
\hline Hauteur $(\mathrm{m})$ & 12,3 & 13,6 & 13,645 & 13,105 \\
\hline $\begin{array}{l}\text { Épaisseur des parois au niveau } \\
\text { du cœur }(\mathrm{m})\end{array}$ & 0,20 & 0,22 & 0,225 & 0,25 \\
\hline
\end{tabular}

\subsubsection{Le circuit primaire et les circuits secondaires}

Le circuit primaire évacue la chaleur dégagée dans le cœur du réacteur grâce à une circulation d'eau sous pression, dite eau primaire, dans les boucles de refroidissement (trois pour un réacteur de $900 \mathrm{MWe}$, quatre pour un réacteur de $1300 \mathrm{MWe}$ ou de $1450 \mathrm{MWe}$ ou encore pour un réacteur de type EPR). Chaque boucle est raccordée à la cuve qui contient le cœur du réacteur et est équipée d'une pompe de circulation, dite pompe primaire, qui assure la circulation de l'eau échauffée au contact des éléments combustibles vers des échangeurs de chaleur, appelés générateurs de vapeur, dans lesquels l'eau primaire transfère son énergie aux circuits secondaires avant de revenir dans le cœur (figures 2.3 et 2.6). Les pompes primaires sont munies de joints d'étanchéité refroidis en permanence par de l'eau sous pression afin d'éviter les fuites d'eau primaire à l'extérieur du circuit primaire.

Les générateurs de vapeur sont des évaporateurs constitués d'un faisceau de tubes en forme d'épingles, avec des dispositifs de séparation d'humidité incorporés dans la partie secondaire. Le fluide primaire passe dans les tubes en U inversé et chauffe l'eau secondaire introduite au-dessus du faisceau tubulaire. La vapeur produite monte à travers les séparateurs d'humidité et sort par le sommet du générateur de vapeur.

Un ballon, appelé pressuriseur, permet l'expansion de l'eau et la maîtrise de la pression du circuit primaire, qui est normalement de 155 bars, afin de maintenir l'eau, chauffée à plus de $300^{\circ} \mathrm{C}$, sous forme liquide. Les réacteurs en exploitation comportent trois lignes de décharge munies chacune d'une soupape d'isolement et d'une soupape de protection. Ces soupapes permettent notamment une dépressurisation ultime du circuit primaire pour éviter une fusion du cœur en pression.

Le pressuriseur du réacteur EPR est quant à lui équipé à son sommet de trois lignes de décharge équipées chacune d'une soupape pilotée munie d'un capteur de position. Le réacteur EPR est équipé en plus d'un dispositif spécifique de dépressurisation ultime du circuit primaire, constitué d'un train de vannes motorisées, destiné à éviter la fusion du cœur en pression. Ce dispositif est constitué de deux lignes de décharge en parallèle connectées à un piquage commun dédié, localisé en haut du pressuriseur. Ces deux 


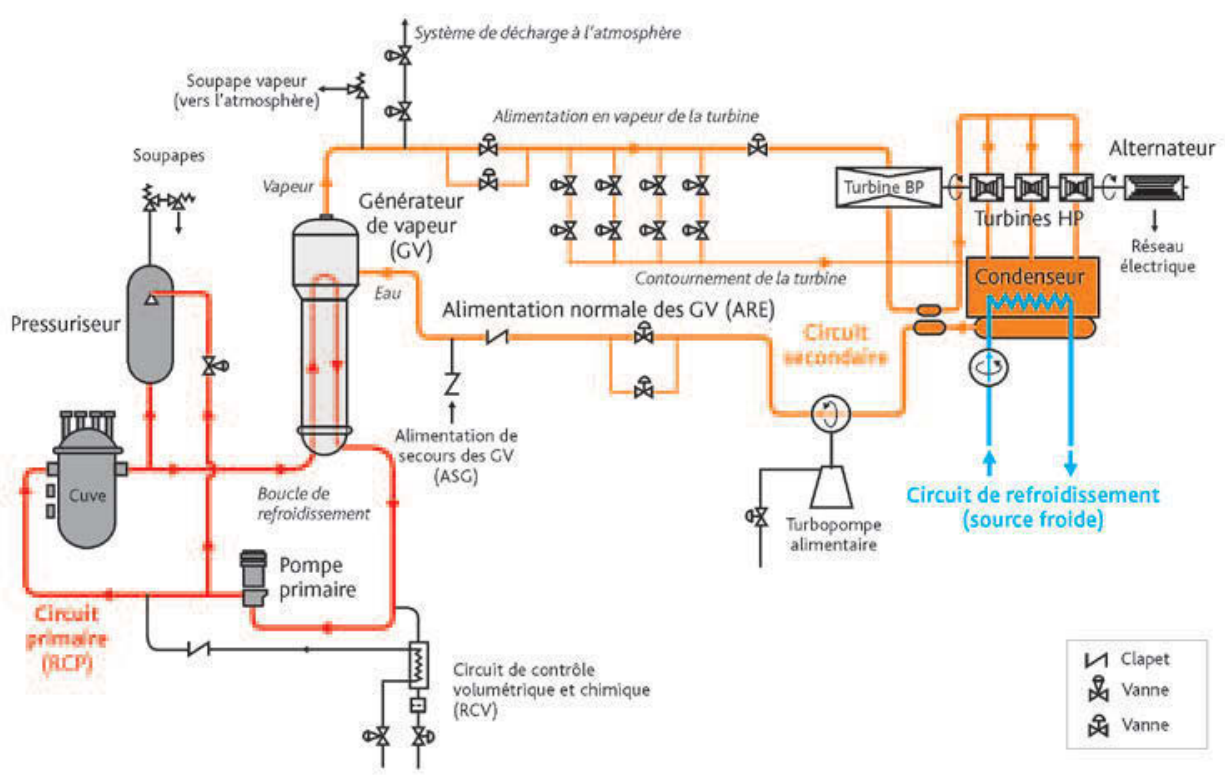

Figure 2.6. Schéma des principaux composants du circuit primaire et des circuits secondaires.

lignes sont munies chacune de deux vannes motorisées et débitent dans une ligne de décharge commune vers le réservoir de décharge du pressuriseur. Ce dispositif est présenté au paragraphe 4.3.4.

Les conditions normales de fonctionnement du circuit primaire des différents paliers sont indiquées dans le tableau 2.3.

Pour chaque tranche, le circuit primaire principal est contenu en totalité dans l'enceinte de confinement.

Tableau 2.3. Conditions de fonctionnement normales du circuit primaire pour les différents paliers.

\begin{tabular}{|l|c|c|c|c|}
\hline \multicolumn{1}{|c|}{ Palier } & $900 \mathrm{MWe}$ & $1300 \mathrm{MWe}$ & $1450 \mathrm{MWe}$ & EPR \\
\hline Nombre de boucles & 3 & 4 & 4 & 4 \\
\hline $\begin{array}{l}\text { Pression primaire absolue } \\
\text { nominale (bar) }\end{array}$ & 155 & 155 & 155 & 27195 \\
\hline Débit nominal $\left(\mathrm{m}^{3} / \mathrm{h}\right)$ & 21250 & 23325 & 24500 & 460 \\
\hline $\begin{array}{l}\text { Volume du circuit primaire y } \\
\text { compris le pressuriseur }\left(\mathrm{m}^{3}\right)\end{array}$ & 271 & 399 & 406 & 296 \\
\hline $\begin{array}{l}\text { Température nominale de } \\
\text { l'eau à l'entrée de la cuve }\left({ }^{\circ} \mathrm{C}\right)\end{array}$ & 286 & 293 & 292 & 330 \\
\hline $\begin{array}{l}\text { Température nominale de } \\
\text { l'eau à la sortie de la cuve }\left({ }^{\circ} \mathrm{C}\right)\end{array}$ & 323 & 329 & 330 & \\
\hline
\end{tabular}


En fonctionnement normal, les circuits secondaires sont utilisés pour convertir l'énergie thermique produite par le cœur en énergie électrique. Afin d'éviter tout transfert d'eau radioactive à l'extérieur de l'enceinte de confinement, les circuits secondaires sont séparés du circuit primaire par les tubes des générateurs de vapeur. Dans ceuxci, l'eau du circuit primaire transmet sa chaleur à l'eau des circuits secondaires qui est vaporisée puis se détend dans la turbine à vapeur de l'installation couplée à l'alternateur (figure 2.6). La vapeur produite dans ces circuits est à une pression de 58 bars absolus pour les réacteurs de $900 \mathrm{MWe}$, de 65 bars absolus pour les réacteurs de $1300 \mathrm{MWe}$, de 73 bars absolus pour les réacteurs de $1450 \mathrm{MWe}$ et de 77 bars absolus pour EPR. À la sortie de la turbine, la vapeur passe dans un condenseur refroidi par l'eau d'une rivière ou d'un fleuve ou par l'eau de la mer, ou par l'intermédiaire d'aéroréfrigérants où l'eau est refroidie au contact de l'air.

Trois ou quatre lignes ${ }^{8}$ (une ligne par générateur de vapeur) relient la partie supérieure des générateurs de vapeur au barillet « vapeur » de la turbine (figure 2.6). Chaque ligne est équipée :

- d'un limiteur de débit placé dans la tubulure de sortie du générateur de vapeur ;

- d'un système de décharge à l'atmosphère équipé d'une vanne d'isolement et d'une vanne de régulation ;

- de sept (deux pour le palier N4 et le réacteur EPR) soupapes de sûreté avec des tuyauteries de décharge à l'atmosphère ;

_ d'une vanne d'isolement qui se ferme en quelques secondes.

Le limiteur de débit permet de réduire la vitesse de refroidissement et de dépressurisation du circuit secondaire concerné et les efforts subis par le faisceau tubulaire correspondant en cas de rupture d'une tuyauterie de vapeur. Les soupapes protègent le circuit contre les surpressions si le débit de vapeur ne peut plus être évacué. Le système de contournement permet d'envoyer momentanément de la vapeur directement au condenseur en contournant la turbine sans solliciter les soupapes. Il est notamment utilisé pour évacuer la chaleur du cœur lors d'un démarrage, d'un arrêt à chaud ou d'un arrêt à froid du réacteur jusqu'à la mise en service du circuit de refroidissement à l'arrêt du réacteur (RRA) (figure 2.7). Le système de décharge à l'atmosphère permet d'évacuer la chaleur résiduelle et donc de refroidir le cœur du réacteur si celui-ci ne peut plus être refroidi par les moyens normaux et de ne pas solliciter les soupapes de sûreté en cas de rupture d'un ou plusieurs tubes de générateur de vapeur. Ce système est constitué d'une seule ligne par générateur de vapeur pour les paliers 900 et $1300 \mathrm{MWe}$, de deux lignes par générateur de vapeur pour le palier N4 et d'une seule ligne pour le réacteur EPR. Chaque ligne est équipée d'une vanne de décharge et d'une vanne d'isolement.

Les caractéristiques des circuits secondaires sont précisées pour les différents paliers dans le tableau 2.4 .

8. Pour le réacteur EPR, chacune des quatre lignes est dans un local séparé. 
Tableau 2.4. Caractéristiques des circuits secondaires pour les différents paliers.

\begin{tabular}{|l|c|c|c|c|}
\hline \multicolumn{1}{|c|}{ Palier } & $900 \mathrm{MWe}$ & $1300 \mathrm{MWe}$ & $1450 \mathrm{MWe}$ & EPR \\
\hline $\begin{array}{l}\text { Nombre de générateurs } \\
\text { de vapeur (GV) }\end{array}$ & 3 & 4 & 4 & 4 \\
\hline $\begin{array}{l}\text { Pression de la vapeur secon- } \\
\text { daire à la sortie des GV (bars } \\
\text { absolus) }\end{array}$ & 58 & 65 & 73 & 77 \\
\hline $\begin{array}{l}\text { Surface d'échange dans un } \\
\mathrm{GV}\left(\mathrm{m}^{2}\right)\end{array}$ & 4746 & 6940 & 7308 & 7960 \\
\hline Débit de vapeur (t/h) par GV & 1820 & 1909 & 2164 & 2197 \\
\hline $\begin{array}{l}\text { Température de la vapeur à la } \\
\text { sortie du GV }\left({ }^{\circ} \mathrm{C}\right)\end{array}$ & 273 & 281 & 288 & 293 \\
\hline
\end{tabular}

\subsubsection{L'enceinte de confinement}

L'enceinte de confinement est constituée du bâtiment du réacteur qui abrite le circuit primaire, une partie des circuits secondaires, incluant les générateurs de vapeur, ainsi que certains auxiliaires de fonctionnement et de sûreté. Le bâtiment du réacteur est constitué d'un cylindre en béton, surmonté d'un dôme en béton, et constitue une enveloppe résistante et à étanchéité spécifiée (voir le chapitre 6 pour plus de détails) ; il assure le confinement des substances radioactives par rapport à l'environnement extérieur et la protection du réacteur contre les agressions externes. Il est conçu, pour les REP en exploitation, pour résister à la pression atteinte ( 4 à 5 bars absolus) lors d'un accident de perte de réfrigérant primaire (APRP avec une rupture complète doublement débattue d'une tuyauterie primaire principale) ou lors d'une rupture d'une tuyauterie de vapeur à l'intérieur de l'enceinte de confinement; il assure une étanchéité satisfaisante dans ces circonstances. Pour le réacteur EPR, il est conçu pour résister à une pression supérieure, de l'ordre de 6,5 bars absolus.

Pour tous les réacteurs, les parois en béton reposent sur un radier lui-même en béton qui constitue le socle du bâtiment et sont coiffées d'un dôme également en béton qui constitue le toit du bâtiment. Le bâtiment du réacteur est dimensionné pour résister aux sollicitations du séisme majoré de sécurité (SMS) (la magnitude du SMS est définie à partir des magnitudes des séismes maximaux historiquement vraisemblables (SMHV) en prenant une marge de sécurité couvrant en particulier les incertitudes) et aux agressions de l'environnement (conditions météorologiques extrêmes, chutes d'avions, explosions, etc.).

Les pénétrations du bâtiment du réacteur constituent des points singuliers de l'enceinte. Des orifices, ou traversées dans les parois de l'enceinte, permettent en effet le passage de tuyauteries, de câbles électriques et des conduits de ventilation. De plus, des sas sont installés pour permettre le passage du personnel et du matériel (gros équipements). Enfin, il existe un tube de transfert des assemblages combustibles entre le bâtiment du réacteur et le bâtiment du combustible. Certaines tuyauteries d'eau et de vapeur constituent une extension de l'enceinte de confinement, notamment les portions 
des circuits secondaires intérieures au bâtiment du réacteur et les portions extérieures jusqu'aux vannes d'isolement. L'enveloppe secondaire des générateurs de vapeur et les faisceaux de tubes des générateurs de vapeur qui font partie du circuit primaire principal sont également une extension de l'enceinte de confinement.

Toutes ces traversées sont à étanchéité spécifiée (voir le chapitre 6 pour plus de détails). Elles sont munies d'organes d'isolement à l'intérieur de l'enceinte, à l'exception des traversées d'eau et de vapeur des circuits secondaires. Ces dispositifs d'isolement, fermés avant un accident ou au cours de celui-ci, sont placés sur les entrées et les sorties de fluide. Pour les traversées d'eau et de vapeur des circuits secondaires, les vannes d'isolement sont placées à l'extérieur de l'enceinte en aval des soupapes de sûreté (voir la description des circuits secondaires au paragraphe précédent).

L'enceinte de confinement fait l'objet de contrôles et d'épreuves avant le premier démarrage du réacteur, notamment pour déterminer son niveau global d'étanchéité et sa résistance aux sollicitations en fonctionnement normal et accidentel. Tous ces aspects sont détaillés dans le chapitre 6 du présent ouvrage.

Des structures internes permettent d'assurer le supportage des matériels, la protection biologique des personnes ainsi qu'une séparation physique des boucles et de certains matériels notamment pour les boucles primaires.

Tableau 2.5. Caractéristiques des enceintes de confinement des différents paliers.

\begin{tabular}{|c|c|c|c|c|c|}
\hline Palier & $900 \mathrm{MWe}$ & $1300 \mathrm{MWe}$ P4 & $1300 \mathrm{MWe} \mathrm{P}^{\prime} 4$ & N4 & EPR \\
\hline Volume total $\left(\mathrm{m}^{3}\right)$ & 60000 & 98000 & 83700 & 86000 & 102700 \\
\hline Volume libre $\left(\mathrm{m}^{3}\right)$ & 50400 & 82000 & 70500 & 73000 & 75500 \\
\hline $\begin{array}{l}\text { Hauteur totale } \\
\text { au-dessus du sol (m) }\end{array}$ & $\begin{array}{l}51,3 \text { (FES)* } \\
52,9 \text { (BUG)* } \\
56,6 \text { (CPY) }\end{array}$ & 71,9 & 61,8 & 63,2 & 62,2 \\
\hline $\begin{array}{l}\text { Diamètre intérieur de la } \\
\text { partie cylindrique }(\mathrm{m})\end{array}$ & 37 & $\begin{array}{c}45,00 \text { (paroi } \\
\text { intérieure) } \\
50,80 \text { (paroi } \\
\text { extérieure) }\end{array}$ & $\begin{array}{c}43,80 \text { (paroi } \\
\text { intérieure) } \\
49,80 \text { (paroi } \\
\text { extérieure) }\end{array}$ & $\begin{array}{c}\text { 43,80 (paroi } \\
\text { intérieure) } \\
49,80 \text { (paroi } \\
\text { extérieure) }\end{array}$ & $\begin{array}{c}48,00 \text { (paroi } \\
\text { intérieure) } \\
53,00 \text { (paroi } \\
\text { extérieure) }\end{array}$ \\
\hline $\begin{array}{l}\text { Épaisseur courante de la } \\
\text { partie cylindrique }(\mathrm{m})\end{array}$ & $\begin{array}{l}0,85(\mathrm{CPO}) \\
0,90 \text { (CPY) }\end{array}$ & $\begin{array}{l}0,90 \text { (paroi } \\
\text { intérieure) } \\
0,55 \text { (paroi } \\
\text { extérieure) }\end{array}$ & $\begin{array}{l}1,20 \text { (paroi } \\
\text { intérieure) } \\
0,55 \text { (paroi } \\
\text { extérieure) }\end{array}$ & $\begin{array}{l}\text { 1,20 (paroi } \\
\text { intérieure) } \\
0,55 \text { (paroi } \\
\text { extérieure) }\end{array}$ & $\begin{array}{l}1,30 \text { (paroi } \\
\text { intérieure) } \\
1,30 \text { (paroi } \\
\text { extérieure) }\end{array}$ \\
\hline $\begin{array}{l}\text { Hauteur hors sol de la } \\
\text { partie cylindrique (m) }\end{array}$ & 41 & $\begin{array}{c}54,15 \text { (paroi } \\
\text { intérieure) } \\
55,04 \text { (paroi } \\
\text { extérieure) }\end{array}$ & $\begin{array}{c}\text { 46,60 (paroi } \\
\text { intérieure) } \\
51,15 \text { (paroi } \\
\text { extérieure) }\end{array}$ & $\begin{array}{c}51,00 \text { (paroi } \\
\text { intérieure) } \\
55,55 \text { (paroi } \\
\text { extérieure) }\end{array}$ & $\begin{array}{l}\text { 43,90 (paroi } \\
\text { intérieure) } \\
\text { 46,60 (paroi } \\
\text { extérieure) }\end{array}$ \\
\hline $\begin{array}{l}\text { Dôme, épaisseur cou- } \\
\text { rante }(\mathrm{m})\end{array}$ & $\begin{array}{l}0,75(\mathrm{CPO}) \\
0,80(\mathrm{CPY})\end{array}$ & $\begin{array}{l}0,87 \text { (paroi } \\
\text { intérieure) } \\
0,40 \text { (paroi } \\
\text { extérieure) }\end{array}$ & $\begin{array}{l}0,82 \text { (paroi } \\
\text { intérieure) } \\
0,40 \text { (paroi } \\
\text { extérieure) }\end{array}$ & $\begin{array}{l}\text { 0,82 (paroi } \\
\text { intérieure) } \\
0,40 \text { (paroi } \\
\text { extérieure) }\end{array}$ & $\begin{array}{l}\text { 1,00 (paroi } \\
\text { intérieure) } \\
1,80 \text { (paroi } \\
\text { extérieure) }\end{array}$ \\
\hline $\begin{array}{l}\text { Épaisseur de la peau } \\
\text { d'étanchéité (mm) }\end{array}$ & 6 & sans peau & sans peau & sans peau & 6 \\
\hline
\end{tabular}

$* \mathrm{FES}=$ Fessenheim $; \mathrm{BUG}=$ Bugey . 
Les enceintes de confinement des réacteurs français, hormis celles des réacteurs de type EPR ${ }^{9}$, sont munies d'un circuit de décompression et de filtration (appelé également dispositif d'éventage-filtration) permettant d'éviter une défaillance brutale de l'enceinte en cas de montée lente de la pression interne lors d'un accident de fusion du cœur. Pour réduire le relâchement de produits radioactifs, la vapeur présente dans l'enceinte est envoyée par ce circuit dans un dispositif comportant un préfiltre métallique et un lit de sable qui permettent de piéger la plus grande partie des aérosols radioactifs. L'ouverture de ce circuit relève d'une procédure spécifique, dénommée procédure U5 (\$ 2.5.2.1).

\section{Description des parois de l'enceinte}

Les enceintes de confinement des réacteurs de 900 MWe sont constituées d'une seule paroi en béton armé précontraint revêtues sur sa surface interne d'une peau d'étanchéité métallique appelée "peau d'étanchéité ». La peau d'étanchéité est chargée d'assurer l'étanchéité de l'enceinte, y compris en cas d'accident. L'enceinte en béton est chargée de résister aux pressions et températures en cas d'accident, aux sollicitations sismiques et aux agressions d'origine externe.

Les enceintes des réacteurs de $1300 \mathrm{MWe}$ et de $1450 \mathrm{MWe}$ comportent deux parois :

- une paroi interne en béton précontraint sans peau d'étanchéité métallique, chargée de résister aux pressions et températures en cas d'accident et participant à l'étanchéité de l'enceinte ;

- une paroi externe en béton armé ; les fuites de la paroi interne sont collectées dans l'espace entre les deux parois (interne et externe) ; cet espace est maintenu en dépression par le système EDE de façon à collecter et à filtrer avant rejet les fuites éventuelles venant de la paroi interne et des traversées. La paroi externe protège également le réacteur des agressions de l'environnement (conditions météorologiques extrêmes, chutes d'avions, explosions, etc.).

L'enceinte du réacteur EPR (Flamanville 3) comporte, comme celles des réacteurs de $1300 \mathrm{MWe}$ et de $1450 \mathrm{MWe}$, deux parois avec un système de confinement dynamique. De plus, la paroi interne de l'enceinte est recouverte d'une peau d'étanchéité métallique. Dans le cas de ce réacteur, l'étanchéité est assurée essentiellement par la peau d'étanchéité. La paroi externe en béton armé de l'EPR a été renforcée par rapport à celle des réacteurs de la génération précédente pour résister, en cas d'accident grave, aux explosions d'hydrogène, répondre aux exigences d'absence directe de fuites radioactives vers l'environnement et consolider sa protection face aux agressions externes.

9. Dans son avis n² 2012-AV-0139 du 3 janvier 2012 relatif aux évaluations complémentaires de sûreté des installations nucléaires prioritaires au regard de l'accident survenu sur la centrale nucléaire de Fukushima Daiichi, l'ASN préconise qu'« EDF devra identifier les systèmes existants ou supplémentaires devant faire partie du « noyau dur » du réacteur EPR en particulier pour assurer la maîtrise de la pression dans l'enceinte en cas « d'accident grave ». Cette préconisation pourrait conduire à reconsidérer la mise en place d'un dispositif d'éventage-filtration sur ce type de réacteur. 


\section{Description du radier de l'enceinte}

Le bâtiment du réacteur repose sur un radier en béton précontraint qui constitue la fondation des parois et des structures internes en béton de l'enceinte de confinement et qui assure le confinement de la partie inférieure du bâtiment. Le radier présente l'aspect d'une galette de béton dont la configuration varie d'un site à l'autre et est adaptée aux caractéristiques sismiques et géotechniques du site considéré. Son épaisseur est de 1,50 m pour Fessenheim, de 2,25 m pour Bugey, de 4,00 m environ pour les tranches CPY, de 3,00 m pour les tranches $\mathrm{P} 4$, de 2,80 m pour les tranches $\mathrm{P}^{\prime} 4$, d'environ $3 \mathrm{~m}$ pour les tranches $\mathrm{N} 4$ et d'environ $4 \mathrm{~m}$ pour les réacteurs de type EPR.

Le radier comporte des galeries de mise en tension des câbles de précontrainte, un réseau de drainage, éventuellement des dispositifs de mesure.

Le radier du réacteur EPR est équipé de plus d'un système de récupération et de refroidissement du corium en cas de défaillance de la cuve lors d'un accident de fusion du cœur. Ce système est présenté dans le détail au paragraphe 4.3.4 du présent ouvrage (voir également la figure 4.7).

\section{- Description du puits de cuve}

Le puits de cuve est limité par un anneau cylindrique en béton entourant la cuve du réacteur. En partie basse, une ouverture est prévue pour permettre l'accès à l'intérieur du puits de cuve (cet accès est condamné lorsque le réacteur est en fonctionnement). L'espace entre la cuve et le béton du puits de cuve est occupé principalement par le calorifuge de la cuve. Le puits de cuve supporte les structures internes du bâtiment du réacteur et repose sur le radier de l'enceinte de confinement (tableau 2.6).

Tableau 2.6. Caractéristiques des puits de cuve des différents paliers.

\begin{tabular}{|l|c|c|c|c|c|}
\hline \multicolumn{1}{|c|}{ Palier } & $900 \mathrm{MWe}$ & $\mathbf{1 3 0 0} \mathrm{MWe}$ P4 & $\mathbf{1 3 0 0} \mathrm{MWe} \mathrm{P}^{\prime} 4$ & $\mathbf{1 4 5 0} \mathrm{MWe}$ 4 & EPR \\
\hline Diamètre interne $(\mathrm{m})$ & 5,20 & 5,85 & 5,26 & 5,56 & 6,15 \\
\hline Épaisseur $(\mathrm{m})$ & 1,80 à 2,10 & 2,00 & 2,00 & 2,00 & 2,70 \\
\hline
\end{tabular}

En partie haute, des saignées permettent le passage des chambres de mesure du flux neutronique et des trémies permettent le passage des tuyauteries primaires (branches chaudes et branches froides).

Le rôle du puits de cuve est de supporter la cuve du réacteur, de protéger les travailleurs des rayonnements ionisants lors des interventions sur le circuit primaire et les matériels avoisinants pendant le fonctionnement.

Pour les réacteurs de 900,1300 et 1450 MWe, le puits de cuve peut contenir de l'eau, notamment lors d'un accident avec une brèche dans le circuit primaire ; l'eau présente dans le puits de cuve peut également provenir de l'utilisation du système d'aspersion dans l'enceinte (§ 2.3.2.4). 
Pour le réacteur EPR, le puits de cuve a été conçu pour éviter la dispersion du corium dans l'enceinte de confinement si une défaillance de la cuve survenait au cours d'un accident de fusion du cœur ; cette disposition vise à éliminer tout risque d'échauffement direct de l'enceinte (§ 5.2.1). Il a également été conçu pour rester sec afin d'éviter une explosion de vapeur qui pourrait résulter d'une interaction corium-eau en cas de défaillance de la cuve ( $§ 5.2 .3$ ) et pour permettre le transfert du corium vers le dispositif d'étalement et de refroidissement spécifique après défaillance de la cuve (§ 5.4.3). La prise en compte des accidents de fusion du cœur dans la conception des réacteurs EPR est présentée en détail au paragraphe 4.3.4.

\subsubsection{Les principaux circuits auxiliaires et circuits de sauvegarde}

Les circuits auxiliaires contribuent, pendant le fonctionnement normal en puissance du réacteur ainsi que lors de la mise à l'arrêt ou du redémarrage normal du réacteur, à l'accomplissement des fonctions fondamentales de sûreté (maîtrise de la réactivité neutronique du cœur, évacuation de la chaleur du circuit primaire et de la puissance résiduelle du combustible, confinement des matières radioactives, voir le paragraphe 2.1). Il s'agit principalement du système de contrôle chimique et volumétrique du réacteur (RCV) et du système de refroidissement du réacteur à l'arrêt (RRA). Ces circuits sont schématisés sur la figure 2.7 qui présente le cas des réacteurs en exploitation (hors EPR).

Le système RCV permet, pendant le fonctionnement du réacteur, d'ajuster la concentration du bore dans l'eau primaire par apport d'eau déminéralisée ou borée en fonction des variations de la puissance du cœur du réacteur. Il est en outre utilisé pour ajuster la masse d'eau dans le circuit primaire en fonction des variations de température. Le circuit RCV permet également de maintenir la qualité de l'eau du circuit primaire, en réduisant sa teneur en produits de corrosion grâce à l'injection de substances chimiques (inhibiteurs de corrosion par exemple). Ce système comporte une ou plusieurs tuyauteries de décharge d'eau en provenance du circuit primaire, un réservoir alimenté en acide borique, une station de purification et une ou plusieurs tuyauteries de charge permettant de réinjecter l'eau dans le circuit primaire. Enfin, ce circuit injecte en permanence de l'eau aux joints des pompes primaires pour assurer leur étanchéité.

Le système RRA a pour fonction, lors d'une mise à l'arrêt normal du réacteur, d'évacuer la chaleur résiduelle produite par le combustible dans la cuve du réacteur et de maintenir l'eau primaire à une température modérée tant qu'il y a du combustible dans le cœur. En effet, après l'arrêt de la réaction en chaîne, le cœur du réacteur continue de produire de la chaleur qu'il est nécessaire d'évacuer pour ne pas risquer d'endommager gravement le combustible. Ce système RRA, qui comporte deux motopompes de circulation, prélève de l'eau dans une boucle primaire à la sortie du cœur, la transfère dans des échangeurs et la renvoie dans une autre boucle primaire à l'entrée du cœur : les échangeurs sont refroidis par le système de réfrigération intermédiaire (RRI), lui-même refroidi par le système d'eau brute secouru (SEC).

Dans le cas du réacteur EPR, le refroidissement à l'arrêt est assuré par le système d'injection de sécurité à basse pression (RIS BP). Le réacteur EPR dispose ainsi de quatre trains séparés et indépendants pour le refroidissement du cœur à l'arrêt. 


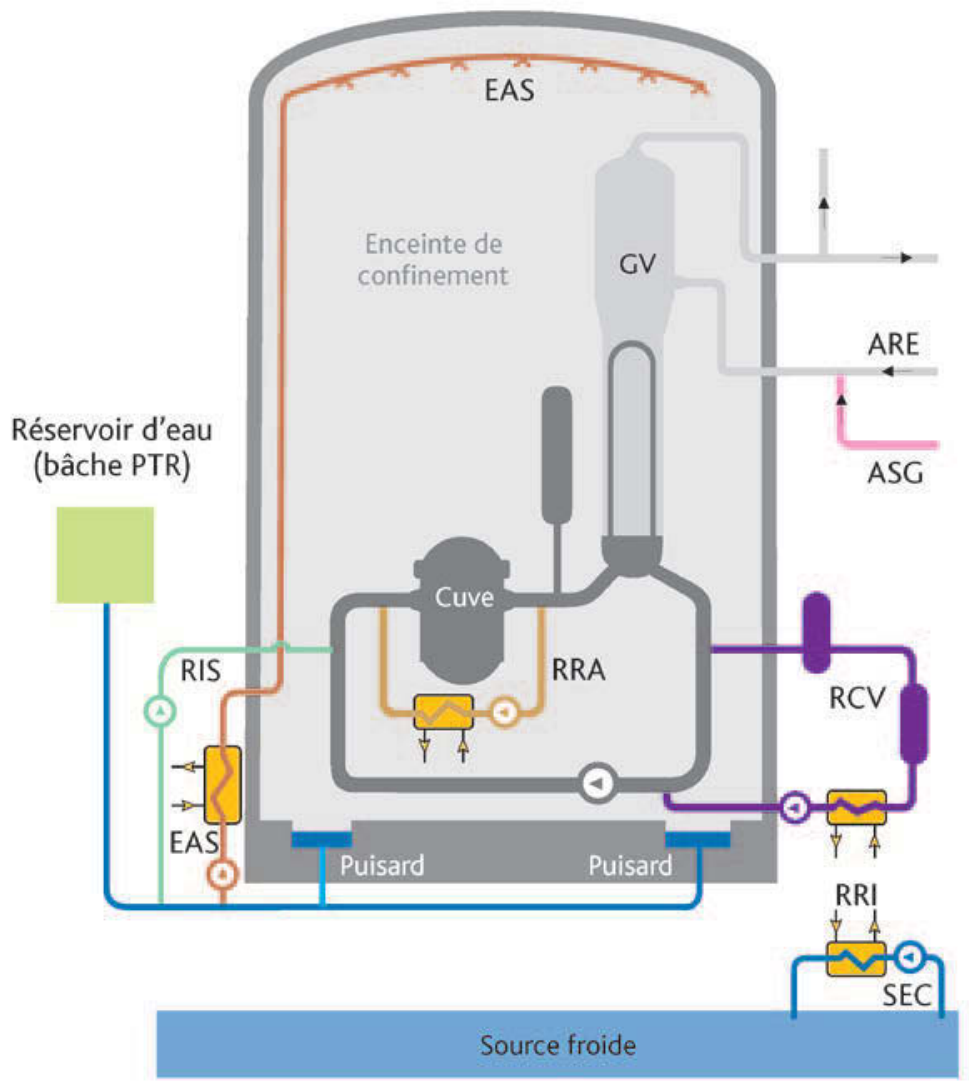

Figure 2.7. Schéma des principaux circuits auxiliaires et des circuits de sauvegarde (réacteurs en exploitation, hors EPR).

Le rôle des systèmes de sauvegarde est de maîtriser les situations accidentelles et d'en limiter les conséquences. Il s'agit principalement du circuit d'injection de sécurité (RIS), du circuit d'aspersion dans l'enceinte (EAS) pour les réacteurs en exploitation (hors EPR) et du circuit d'alimentation de secours en eau des générateurs de vapeur (ASG). Ces circuits sont schématisés sur la figure 2.7 qui présente le cas des réacteurs hors EPR.

Le circuit RIS permet d'injecter de l'eau borée dans le cœur du réacteur en cas d'accident de perte de réfrigérant primaire afin de stopper la réaction nucléaire et de maintenir l'inventaire d'eau dans le circuit primaire. Pour les réacteurs en exploitation (hors EPR), il permet également d'évacuer, dans certains cas de fonctionnement du circuit $^{10}$, la puissance résiduelle.

10. Comme pour le circuit EAS décrit plus loin, le circuit RIS peut injecter de l'eau directement à partir du système de traitement et refroidissement de l'eau des piscines (PTR) ou indirectement en reprenant l'eau récupérée en partie basse de l'enceinte (recirculation). L'évacuation de la puissance résiduelle par le système RIS n'est assurée qu'en mode d'injection direct. En cas de recirculation, la puissance résiduelle est évacuée par le système EAS (§ 2.4.2.2). 
Pour les réacteurs en exploitation (hors EPR), le circuit RIS comporte des accumulateurs sous pression contenant de l'eau borée, un réservoir d'eau borée (bâche PTR) et différentes pompes aux débits et pressions de refoulement adaptés pour répondre aux différents cas d'accident de perte de réfrigérant primaire (différentes tailles de brèches). Les réacteurs en exploitation disposent d'une injection à haute pression et d'une injection à basse pression. Les réacteurs de $1300 \mathrm{MWe}$ disposent en plus d'une injection à pression moyenne.

Le réacteur EPR dispose de quatre trains séparés et indépendants d'injection de sécurité à basse et à moyenne pression. Les quatre trains sont alimentés avec de l'eau borée à partir d'un réservoir (le réservoir IRWST) situé à l'intérieur de l'enceinte de confinement ${ }^{11}$ (contrairement aux réacteurs en exploitation pour lesquels le réservoir d'eau borée est à l'extérieur de l'enceinte de confinement).

Le fonctionnement de ces circuits est décrit au paragraphe 2.4.2.

En cas d'accident conduisant à une augmentation significative de la pression dans le bâtiment du réacteur, un système d'aspersion d'eau (EAS) permet de faire décroître la pression et ainsi de préserver l'intégrité de l'enceinte de confinement. Ce système permet également de rabattre les aérosols radioactifs éventuellement disséminés dans cette enceinte. Pour les réacteurs actuellement en exploitation, le circuit EAS, en partie externe à l'enceinte, permet de pulvériser de l'eau dans le bâtiment du réacteur, soit à partir d'une réserve d'eau externe (PTR), à laquelle on ajoute de la soude, soit en utilisant l'eau récupérée dans la partie basse de l'enceinte (puisard).

Pour les réacteurs de type EPR, le refroidissement de l'enceinte est assuré en cas d'accident grave par la pulvérisation d'eau borée provenant du réservoir IRWST et drainant l'eau à l'intérieur de l'enceinte. Ce système de sauvegarde utilisé uniquement en cas d'accident grave est appellé système CHRS ; son utilisation est également décrite dans le paragraphe 2.4.2.

Le circuit ASG permet de maintenir le niveau d'eau dans la partie secondaire des générateurs de vapeur et donc de refroidir l'eau du circuit primaire en cas d'indisponibilité du système normal d'alimentation (ARE). Pour les réacteurs de 900, 1300 et $1450 \mathrm{MWe}$, il est également utilisé en fonctionnement normal lorsque le réacteur est en arrêt à chaud pour assurer le maintien en eau des générateurs de vapeur. Le système ASG comporte deux pompes entraînées par un moteur électrique et une pompe (900 MWe) ou deux pompes (autres paliers) entraînées par une turbine à vapeur alimentée par les générateurs de vapeur qui puisent l'eau dans une bâche de $700 \mathrm{~m}^{3}$ (900 MWe), $1440 \mathrm{~m}^{3}$ (P4), $1723 \mathrm{~m}^{3}\left(\mathrm{P}^{\prime} 4\right)$ ou $1488 \mathrm{~m}^{3}$ (N4) et l'injectent dans la partie secondaire des générateurs de vapeur.

11. Grâce aux échangeurs de chaleur intégrés à l'injection de sécurité basse pression, l'évacuation de la puissance résiduelle hors de l'enceinte de confinement est assurée pour le réacteur EPR sans avoir à utiliser un système d'aspersion (EAS) comme sur les réacteurs en exploitation. En fait, il existe sur le réacteur EPR un système d'évacuation de la puissance résiduelle, mais il n'est utilisé que pour les situations d'accident grave. Le réservoir IRWST procure également l'eau nécessaire au refroidissement du corium dans le dispositif de récupération dans le cas d'un accident de fusion du cœur avec défaillance de la cuve. 
Dans le cas du réacteur EPR, le système ASG comporte quatre trains séparés et indépendants, chacun possédant son propre réservoir d'eau alimenté par une bâche commune de $2600 \mathrm{~m}^{3}$ et n'est utilisé qu'en cas de défaillance du système d'alimentation normale en eau des générateurs de vapeurs. Le réacteur EPR est également équipé d'un système spécifique d'alimentation en eau des générateurs de vapeur pour le démarrage et l'arrêt du réacteur.

\subsubsection{Les autres systèmes}

Parmi les autres systèmes ou circuits importants pour la sûreté du réacteur, on peut citer :

- le circuit de réfrigération intermédiaire (RRI) qui assure le refroidissement d'un certain nombre d'équipements importants pour la sûreté du réacteur (refroidissement des pompes du circuit primaire et du système de contrôle chimique et volumétrique (RCV), refroidissement des systèmes de ventilation, refroidissement du système d'injection de sécurité (RIS) et du système d'aspersion dans l'enceinte (EAS) pour les tranches en exploitation, refroidissement du système de refroidissement du réacteur à l'arrêt (RRA ou RIS/RRA pour les réacteurs de type EPR). Le circuit RRI fonctionne en boucle fermée entre, d'une part les circuits auxiliaires et les circuits de sauvegarde, d'autre part le circuit d'eau brute secouru (SEC) (voir l'alinéa suivant). Il est à noter que, pour les réacteurs de Fessenheim, le circuit RRI ne participe pas au refroidissement des systèmes RCV, des pompes primaires, du système EAS et des systèmes de ventilation ; le refroidissement de ces systèmes et équipements est assuré directement par le circuit d'eau brute secouru. Tous les autres réacteurs en exploitation sont équipés de deux trains RRI et les réacteurs de type EPR de quatre trains indépendants pour le refroidissement des systèmes de sauvegarde ;

- le circuit d'eau brute secouru (SEC) qui assure le refroidissement du circuit RRI au moyen de la source froide (cours d'eau ou mer). Les paliers en exploitation sont équipés de deux trains SEC, les réacteurs de type EPR de quatre trains;

- le circuit de réfrigération et de purification de l'eau des piscines (PTR) qui permet, entre autres, d'évacuer la chaleur résiduelle des éléments combustibles stockés dans la piscine d'entreposage des combustibles usés ;

- les systèmes de ventilation qui jouent un rôle essentiel dans le confinement des matières radioactives par la mise en dépression des locaux et la filtration des rejets ;

- les circuits et systèmes destinés à la lutte contre l'incendie ;

- le système de contrôle-commande et les systèmes électriques ; l'alimentation électrique des systèmes importants pour la sûreté du réacteur est doublée ; elle comprend pour les réacteurs en exploitation deux voies électriques indépendantes et pour les réacteurs de type EPR quatre voies électriques indépendantes ; chaque voie électrique est alimentée par un tableau électrique, lui-même alimenté, soit par le réseau électrique externe (deux lignes indépendantes à très 
haute tension), soit par un groupe électrogène à moteur diesel. De plus, un troisième groupe électrogène à moteur diesel (palier $900 \mathrm{MWe}$ ) ou une turbine de secours (paliers $1300 \mathrm{MWe}$ et $1450 \mathrm{MWe}$ ) ou deux groupes électrogènes à moteur diesel d'ultime secours (réacteurs de type EPR) peuvent être mis en service en cas de nécessité.

\subsection{Fonctionnement du réacteur en situation normale et en situation accidentelle}

\subsubsection{Les systèmes utilisés lors du fonctionnement normal du réacteur}

Le fonctionnement normal du réacteur recouvre les différents états suivants :

- les états en puissance et l'arrêt à chaud, pour lesquels le refroidissement est assuré par les générateurs de vapeur alimentés du côté secondaire par de l'eau provenant du système normal d'alimentation des générateurs de vapeur (ARE) ;

- les états à l'arrêt, circuit primaire fermé, pour lesquels le refroidissement est assuré, soit par le système de refroidissement à l'arrêt (RRA), soit par les générateurs de vapeur alimentés du côté secondaire par de l'eau provenant du système d'alimentation de secours des générateurs de vapeur (ASG) ;

- les états à l'arrêt, circuit primaire ouvert, pour lesquels le refroidissement est assuré par le système RRA.

Les fonctions fondamentales de sûreté doivent être assurées en permanence pour les différents états du réacteur, à savoir la maîtrise de la réactivité, l'évacuation de la puissance et le confinement des produits radioactifs. Dans la suite, sont décrits les systèmes utilisés pour assurer chacune de ces fonctions.

\subsubsection{Systèmes utilisés pour la maîtrise de la réactivité}

La maîtrise de la réactivité est assurée, d'une part, par de l'acide borique en solution dans l'eau du circuit primaire (le bore est un absorbeur efficace de neutrons) permettant de compenser les évolutions de réactivité à long terme. Elle est assurée, d'autre part, par des grappes de commande ( $(2.3 .2 .1)$ constituées de 24 crayons en acier inoxydable contenant un alliage argent-indium-cadmium (tous les réacteurs) ou du carbure de bore (réacteurs de $1300 \mathrm{MWe}$ et de $1450 \mathrm{MWe}$, réacteur EPR), coulissant dans des tubesguides en zircaloy. Ces grappes s'insèrent plus ou moins dans le cœur permettant la maîtrise de la puissance par les systèmes de régulation de l'installation ou par les opérateurs de la centrale via des commandes manuelles.

Entre l'arrêt à chaud et l'arrêt à froid du réacteur, le coefficient de température étant négatif, la réactivité du cœur du réacteur augmente du fait de la baisse de température (§ 2.1). Les barres de commande ne suffisent pas pour maîtriser cette augmentation de 
la réactivité qui est maîtrisée par une augmentation de la concentration de bore dans le circuit primaire.

L'injection d'acide borique dans le circuit primaire en fonctionnement ou à l'arrêt est assurée par le système de contrôle chimique et volumétrique (RCV ; voir la description de ce système au § 2.3.2.4).

\subsubsection{Systèmes utilisés pour l'évacuation de la puissance}

Lors du fonctionnement en puissance, l'évacuation de l'énergie dégagée dans le cœur du réacteur est réalisée par le circuit primaire qui transfère cette énergie aux générateurs de vapeur grâce aux pompes primaires. La vapeur produite dans la partie secondaire des générateurs de vapeur se détend dans une turbine à vapeur et se condense dans le condenseur, lui-même refroidi par un circuit tertiaire avec une source froide constituée par la mer ou un cours d'eau (circuit ouvert) ou l'atmosphère (via des aéroréfrigérants (circuit fermé)). L'eau du condenseur est renvoyée dans les générateurs de vapeur par le système normal d'alimentation en eau des générateurs de vapeur (ARE) (figures 2.3 et 2.6).

Lorsque le réacteur est à l'arrêt, la puissance résiduelle due aux produits de fission est beaucoup plus faible (inférieure à quelques pour cent de la puissance nominale) et décroissante en fonction du temps; elle est évacuée par différents moyens en fonction de l'état ouvert ou fermé du circuit primaire. Lorsque le circuit primaire est fermé, l'énergie dégagée peut être transférée aux générateurs de vapeur par convection naturelle sans l'aide des pompes primaires. L'alimentation en eau des générateurs de vapeur peut être faite, soit par le système ARE, soit par le système d'alimentation de secours (ASG). Lorsque le système ASG est utilisé, la vapeur produite est rejetée dans l'atmosphère par l'intermédiaire de vannes réglables (figure 2.6).

Un autre moyen d'évacuer la puissance résiduelle est constitué, pour les réacteurs en exploitation (hors EPR), par le système de refroidissement à l'arrêt (RRA) qui peut remplir cette fonction lorsque la cuve est fermée ou lorsque la cuve est ouverte (voir la description de ce système au § 2.3.2.4, figure 2.7).

Dans le cas du réacteur EPR, la fonction de refroidissement du réacteur à l'arrêt est assurée par le système d'injection de sécurité à basse pression (§ 2.3.2.4).

\subsubsection{Systèmes utilisés pour le confinement des produits radioactifs}

En fonctionnement normal, l'eau du circuit primaire contient une faible quantité de produits radioactifs constitués par des produits de corrosion du circuit primaire activés lors de leur passage dans le cœur du réacteur et des produits de fission gazeux ou sous forme de particules provenant de fuites de gaines de crayons combustibles.

L'eau du circuit primaire est purifiée en permanence par le système RCV : les particules sont arrêtées dans des filtres et les produits gazeux entreposés dans des réservoirs pour être traités ultérieurement. 
Afin d'éviter des relâchements de produits radioactifs dans l'environnement dus à des fuites de circuits contenant des produits radioactifs, des dispositions sont mises en place pour en assurer le confinement :

- les locaux ou bâtiments susceptibles de contenir des produits radioactifs sous formes gazeuses ou sous forme d'aérosols sont mis en dépression par rapport à l'extérieur ; les locaux à « risque iode » sont eux-mêmes en dépression par rapport aux locaux qui les entourent ;

- les fuites gazeuses sont récupérées par des circuits spécifiques (notamment, le circuit de traitement des effluents gazeux) pour entreposage et contrôle avant rejet ou par les circuits de ventilation du bâtiment des auxiliaires nucléaires équipés de filtres et pièges à iode ;

- les fuites liquides sont récupérées par des puisards, des cuvelages, des bacs de rétention et par des circuits de collecte pour être traitées et contrôlées avant entreposage dans des réservoirs prévus à cet effet.

Pour le réacteur EPR, des dispositions de conception ont été mises en œuvre pour éviter toute fuite directe de substances radioactives vers l'environnement. Les traversées de l'enceinte de confinement débouchent toutes dans des bâtiments ventilés et équipés de moyens de filtration.

Les mesures de radioactivité effectuées dans les locaux contenant les circuits auxiliaires (activité de l'air ambiant, activité de l'eau des puisards) permettent de surveiller l'étanchéité de ces circuits; les mesures de radioactivité dans le circuit de réfrigération RRI et dans le circuit de vapeur permettent de surveiller l'étanchéité des échangeurs.

\subsubsection{Les systèmes utilisés lors d'un fonctionnement incidentel ou accidentel du réacteur}

$\mathrm{Si}$, lors du fonctionnement normal de l'installation (incluant les régimes transitoires normaux d'exploitation), les paramètres physiques essentiels de l'installation restent dans des plages de valeurs fixées, lors d'un fonctionnement accidentel, certains de ces paramètres peuvent sortir de ces plages, ce qui conduit à la mise en œuvre de systèmes (protection, sauvegarde, voir $\S 2.3 .2 .4$ et $\S 2.3 .2 .5$ ) prévus à la conception pour pouvoir ramener le réacteur dans un état assurant les trois fonctions fondamentales de sûreté : maîtrise de la réactivité, évacuation de la puissance et confinement des produits radioactifs.

\subsubsection{Systèmes utilisés pour la maîtrise de la réactivité}

La maîtrise de la réactivité est réalisée grâce à l'insertion dans le cœur du réacteur de l'ensemble des barres de commande ${ }^{12}$ qui chutent par gravité en deux à trois secondes en cas de coupure du circuit électrique (arrêt automatique). Les valeurs de certains

12. La défaillance d'un mécanisme de commande peut éventuellement conduire à l'éjection d'une barre de commande et à une augmentation non maîtrisée de la réactivité dans l'assemblage concerné. Ce type d'accident de réactivité fait l'objet d'études et de recherches importantes, notamment dans le cadre du programme international CABRI de l'IRSN ; il n'est pas traité dans le cadre de cet ouvrage. 
paramètres sont comparées en permanence à des seuils (puissance du réacteur, pression du circuit primaire, vitesse des pompes primaires, températures, etc.) ; lorsqu'un de ces seuils est franchi, le système de protection déclenche l'arrêt automatique et éventuellement d'autres actions : lors de certaines situations accidentelles, le système d'injection de sécurité est également mis en service pour maîtriser la réactivité par injection d'eau borée dans le circuit primaire ; le système RCV peut également compenser les pertes d'eau borée du circuit primaire en cas de fuite limitée.

\subsubsection{Systèmes utilisés pour l'évacuation de la puissance résiduelle}

Dans les situations accidentelles où le circuit primaire reste intègre, l'évacuation de la puissance résiduelle peut être réalisée dans un premier temps par le système d'alimentation de secours en eau des générateurs de vapeur (ASG) qui démarre automatiquement s'il fonctionne en cas de perte de l'alimentation normale en eau des générateurs de vapeur (figure 2.7).

Dans certaines situations accidentelles, l'intégrité du circuit primaire est mise en défaut, soit par une défaillance du circuit, soit par l'ouverture de soupapes de sécurité. L'évacuation de la puissance résiduelle ne peut alors être assurée que si, d'une part l'inventaire d'eau dans le circuit primaire reste suffisant, d'autre part l'énergie dégagée dans l'enceinte de confinement par le fluide sortant par la brèche du circuit primaire est évacuée. Par exemple, dans les situations de petite brèche dans le circuit primaire, l'énergie dégagée par le cœur du réacteur n'est pas entièrement évacuée par le fluide sortant par la brèche dans l'enceinte et il est nécessaire d'évacuer une partie de cette énergie par le système ASG.

\section{- Maintien de l'inventaire d'eau dans le circuit primaire}

Le maintien d'un inventaire d'eau suffisant dans le circuit primaire est assuré par le système d'injection de sécurité (RIS) qui est capable d'injecter de l'eau en quantité suffisante dans le circuit primaire pour des tailles de brèches dans ce circuit allant jusqu'à la rupture complète d'une tuyauterie principale.

Pour les réacteurs de $900 \mathrm{MWe}$, cette mission est assurée pour l'ensemble des brèches par deux pompes pouvant injecter de l'eau borée à haute pression (seuil de déclenchement à 170 bars) et deux pompes pouvant injecter de l'eau borée à basse pression (seuil de déclenchement à 10 bars). De plus, des accumulateurs remplis d'eau borée et maintenus sous pression d'azote se vident dans le circuit primaire dès que la pression dans ce circuit devient inférieure à 40 bars. Pour les réacteurs de $1300 \mathrm{MWe}$ et de $1450 \mathrm{MWe}$, il existe une injection à moyenne pression (deux pompes permettant d'injecter de l'eau borée dans les branches « froides » avec un seuil de déclenchement à 120 bars), une injection à basse pression (deux pompes permettant l'injection d'eau borée dans les branches « froides » et dans les branches " chaudes » avec un seuil de déclenchement à 20 bars) et quatre accumulateurs qui se vident dans le circuit primaire dès que la pression dans ce circuit devient inférieure à 40 bars. 
Pour les réacteurs en exploitation (hors EPR), le système RIS est automatiquement mis en service par le système de protection dès lors que la pression mesurée dans le pressuriseur du circuit primaire devient faible (seuils de déclenchement indiqués plus haut pour les différents paliers). Dans la première phase de fonctionnement du système RIS, l'eau injectée dans le circuit primaire provient d'une réserve d'eau borée : le réservoir de stockage de l'eau des piscines (dit bâche PTR, implanté à l'extérieur du bâtiment du réacteur). Quand cette réserve est épuisée, le système fonctionne grâce à l'EAS en recirculation en circuit fermé en utilisant l'eau provenant de la condensation de la vapeur dans l'enceinte qui s'est écoulée dans des puisards situés en partie basse de celle-ci.

Le système d'injection de sécurité est également capable de maintenir un inventaire d'eau suffisant dans le circuit primaire dans des situations accidentelles où le circuit primaire reste intègre et où l'évacuation de la puissance par les générateurs de vapeur est impossible. Dans ces situations, l'ouverture volontaire des soupapes de sécurité du circuit primaire et la mise en service du système d'injection de sécurité permettent d'assurer le maintien d'un inventaire d'eau suffisant, d'éviter la mise en surpression du circuit primaire et d'évacuer la puissance résiduelle (fonctionnement en " gavé-ouvert »). L'eau injectée à basse température (de l'ordre de quelques dizaines de ${ }^{\circ} \mathrm{C}$ ) par le système d'injection de sécurité traverse le cœur et ressort sous forme de vapeur par les soupapes.

Pour EPR, il existe une injection à moyenne pression (quatre pompes permettant d'injecter de l'eau borée dans les branches « froides » avec un seuil de déclenchement à environ 90 bars), une injection à basse pression (quatre pompes permettant l'injection d'eau borée dans les branches «froides » et dans les branches « chaudes » avec un seuil de déclenchement à 20 bars) et quatre accumulateurs qui se vident dans le circuit primaire dès que la pression dans ce circuit devient inférieure à 40 bars. L'eau injectée dans le circuit primaire provient du réservoir d'eau borée IRWST situé à l'intérieur de l'enceinte de confinement.

\section{- Évacuation de l'énergie dégagée dans l'enceinte de confinement}

Pour les réacteurs en exploitation (hors EPR), en cas de brèche dans le circuit primaire, l'évacuation de l'énergie hors de l'enceinte de confinement et la décroissance de la pression dans cette enceinte sont assurées par le système d'aspersion dans l'enceinte (EAS) qui comporte deux motopompes aspirant à court terme l'eau de la bâche PTR jusqu'à son épuisement, puis à plus long terme celle récupérée dans les puisards de l'enceinte (tous paliers). L'eau de ce circuit est refroidie par le système de refroidissement intermédiaire (RRI), refroidi lui-même par le système d'eau brute secouru (SEC) (pour Fessenheim, le refroidissement est assuré directement par le système d'eau brute secouru (SEC)) (figure 2.7).

Dans certaines situations d'accident avec fusion du cœur menaçant l'intégrité de l'enceinte, l'énergie peut être évacuée par le circuit d'éventage-filtration de l'enceinte de confinement qui permet d'écrêter la pression dans l'enceinte (procédure U5; $\S$ 2.5.2.1). 
Pour le réacteur EPR, en cas de brèche dans le circuit primaire, l'évacuation de l'énergie hors de l'enceinte de confinement et la décroissance de la pression dans cette enceinte sont assurées par le système d'injection de sécurité à basse pression via les échangeurs de chaleur du circuit RIS/RRA qui comporte quatre motopompes aspirant l'eau du réservoir IRWST.

Un système spécifique, le système CHRS, a été mis en place pour évacuer l'énergie hors de l'enceinte de confinement et faire décroître la pression dans cette enceinte lors d'un accident de fusion du cœur. Ce système comprend un système d'aspersion à deux trains, des échangeurs et une source froide spécifique.

Lors d'un accident de fusion du cœur avec défaillance de la cuve, l'eau du réservoir IRWST sert à noyer et refroidir le corium dans le dispositif de récupération de celui-ci. Le système CHRS, système d'aspersion dédié aux accidents graves, permet d'alimenter la circulation d'eau dans ce dispositif et de réduire la vaporisation de l'eau recouvrant le corium et la montée en pression de l'enceinte de confinement.

\subsubsection{Systèmes utilisés pour le confinement des produits radioactifs}

En situation accidentelle, les gaines peuvent perdre leur étanchéité parce que la puissance dégagée dans les crayons combustibles n'est pas correctement évacuée ; l'élévation de la température conduit au fluage puis à l'éclatement des gaines et à des réactions d'oxydation du zirconium de ces gaines par la vapeur d'eau.

Pour les situations incidentelles de fréquence estimée relativement élevée susceptibles de se produire durant la vie du réacteur, le confinement des produits de fission reste principalement assuré par les gaines des crayons combustibles qui sont conçues pour rester étanches dans ces situations. L'enveloppe du circuit primaire et l'enceinte de confinement constituent deux barrières supplémentaires assurant le confinement des produits de fission en cas de défaillance des gaines de crayons combustibles ainsi que des produits d'activation présents dans l'eau du circuit primaire.

Pour les situations de plus faible fréquence estimée pour lesquelles les gaines et l'enveloppe du circuit primaire ne sont plus étanches, le confinement est assuré par le bâtiment du réacteur qui est conçu pour maintenir une bonne étanchéité. Pour éviter les fuites par les circuits traversant l'enceinte de confinement, des vannes et des clapets d'étanchéité sont disposés sur toutes les traversées de l'enceinte ; les vannes sont automatiquement fermées dès que la pression dans l'enceinte dépasse un seuil fixé (isolement de l'enceinte). Ces dispositions visent à assurer un très faible taux de fuite de l'enceinte de confinement vers l'atmosphère.

Il existe une autre source de rejets radioactifs constituée par les produits radioactifs (essentiellement les produits de fission) véhiculés par l'eau circulant dans le circuit primaire et les circuits d'injection de sécurité (RIS) et d'aspersion dans l'enceinte (EAS pour les réacteurs en exploitation et CHRS en cas d'accident grave pour le réacteur EPR) en situation accidentelle. Afin de réduire les rejets dans l'atmosphère pouvant survenir en 
cas de fuite de ces circuits, qui sont implantés partiellement hors du bâtiment du réacteur, les bâtiments dans lesquels ils se trouvent sont maintenus en dépression grâce à des systèmes de ventilation munis de dispositifs de filtration.

Le confinement des produits radioactifs doit ainsi être assuré dans toute situation qui, compte tenu de certaines dégradations d'équipements, entraîneraient un contact direct de fluide primaire avec l'extérieur de l'enceinte de confinement, soit dans des bâtiments périphériques, soit directement dans l'environnement. Ces situations, dites de «bipasse » de l'enceinte de confinement, sont décrites en détail dans le paragraphe 6.4. Citons notamment le cas de ruptures de tubes de générateurs de vapeur qui conduit à un transfert de fluide primaire dans les circuits secondaires et peut conduire à des rejets radioactifs dans l'atmosphère par ouverture des vannes de décharge et des soupapes de sûreté de ces générateurs de vapeur ; la prévention des risques associés à ces ruptures de tubes est réalisée par des contrôles périodiques de l'état des tubes, par la mise en place de bouchons pour isoler les tubes fragilisés ou corrodés, par le remplacement des générateurs de vapeur s'il s'avère nécessaire, par la maîtrise de la chimie et de l'activité de l'eau primaire et par des procédures de conduite visant à éviter la sollicitation des organes de décharge à l'atmosphère des générateurs de vapeur.

\subsection{La conduite du réacteur en fonctionnement normal et en situation accidentelle}

\subsubsection{Salle de commande}

La conduite de l'installation est réalisée par une équipe de conduite à partir d'une salle de commande où sont regroupés tous les organes de commande, de signalisation et de surveillance nécessaires au fonctionnement du réacteur en exploitation normale et à sa conduite lors des situations incidentelles et accidentelles.

Après l'accident de TMI-2 aux États-Unis (§7.1), les salles de commande des tranches en exploitation (réacteurs de $900 \mathrm{MWe}$ et de $1300 \mathrm{MWe}$ ) ont été modifiées pour améliorer leur ergonomie avec une disposition et une présentation plus claires et plus fiables des commandes et des informations qui y sont présentées. Une présentation plus précise sur les pupitres de commande de l'état des systèmes permettant de piloter le réacteur et de maîtriser son fonctionnement a ainsi été recherchée. Un outil d'aide à l'application des procédures incidentelles et accidentelles a été installé dans chaque salle de commande : il s'agit du «panneau de sûreté ». Couplé au système d'acquisition de données, cet outil fournit une synthèse des paramètres importants pour l'application des procédures et la conduite de l'installation. Il permet également de connaître rapidement la disponibilité des systèmes importants pour la sûreté : systèmes d'isolement de l'enceinte de confinement, systèmes de sauvegarde, alimentations électriques, etc. Lors d'un accident, il fournit aux opérateurs des informations mieux adaptées (état des systèmes, niveau d'eau et marge à l'ébullition dans le cœur, étanchéité du confinement, etc.). 
La conduite du réacteur est centralisée en salle de commande tant qu'elle reste accessible au personnel. Si la salle de commande principale est indisponible, notamment si elle doit être évacuée du fait d'un sinistre (par exemple un incendie), un panneau de repli, situé dans un local extérieur à la salle de commande qui doit rester accessible en cas d'incendie dans celle-ci, permet, dans certaines conditions, de procéder à l'arrêt de la tranche et de maintenir le réacteur dans un état sûr. Les commandes du panneau de repli deviennent alors prioritaires sur celles de la salle de commande principale. Il existe également des panneaux de commande décentralisés qui concernent des fonctions particulières : traitement des effluents, déminéralisation de l'eau, commande locale des groupes électrogènes à moteur diesel, etc.).

Toutes les tranches sont également équipées d'un local de crise. Ce local est mis à disposition de l'équipe de crise constituée sur le site lors d'un accident. L'équipement de ce local permet à l'équipe de crise locale de prendre connaissance des principaux paramètres de la tranche et de les communiquer aux autres équipes de crise constituées au niveau national qui disposent déjà d'informations sur la tranche concernée. Les équipes de crise (locale et nationales) disposent ainsi d'une connaissance identique des paramètres de la situation, permettant le traitement de l'accident.

Les salles de commande du palier N4 diffèrent des salles de commande des paliers précédents par une utilisation importante de systèmes informatisés. Pour ces derniers réacteurs, les procédures de conduite sont affichées sur écran, les traitements logiques et les surveillances sont automatisés. Un moyen de secours supplémentaire, le panneau auxiliaire, a été prévu en salle de commande pour pallier la perte du système informatique de conduite. Le panneau auxiliaire a pour fonction de permettre d'assurer le repli sûr de la tranche et la maîtrise des situations accidentelles si le système informatique est défaillant. Les salles de commande des réacteurs de type EPR reprennent, d'un point de vue technologique, les choix retenus pour le palier N4.

\subsubsection{Conduite du réacteur}

\subsubsection{Les procédures de conduite}

Durant le fonctionnement normal de l'installation et pour les transitoires incidentels et accidentels, la conduite utilise un ensemble de procédures de conduite qui visent à maintenir ou à amener le réacteur dans un état sûr.

Chaque événement initiateur pouvant conduire à un incident ou à un accident est associé à une conduite type, décrite dans les procédures de conduite «I » (pour incidentelles) et «A » (pour accidentelles). Ces procédures sont élaborées à partir du déroulement prévisible de l'incident ou de l'accident afin de maintenir ou de ramener le réacteur dans un état sûr; elles sont applicables si l'événement est unique (pas de cumul) et s'il a été correctement diagnostiqué. Cette approche est appelée « l'approche par événement $»$.

Pour les conditions de fonctionnement avec défaillances simultanées de voies redondantes de systèmes importants pour la sûreté ou pour les cas de défaillances 
de matériels sollicités sur le long terme (pendant plusieurs mois) après un accident de perte de réfrigérant primaire, des procédures complémentaires, dites " procédures $\mathrm{H}$ » (H pour hors dimensionnement), ont été élaborées, nécessitant éventuellement l'installation de nouveaux matériels appelés " matériels complémentaires » (par exemple l'adjonction d'un turbogénérateur fournissant, à partir de la vapeur du circuit secondaire, une source d'énergie électrique alimentant certains systèmes vitaux, ou la mise en place d'un groupe électrogène spécifique (pour les réacteurs de $900 \mathrm{MWe}$ ) ou d'une turbine à combustion (pour les réacteurs de $1300 \mathrm{MWe}$ et de $1450 \mathrm{MWe})$ ). Ces procédures sont les suivantes :

- la procédure $\mathrm{H1}$ pour la défaillance totale de la source froide ou des systèmes associés ;

- la procédure H2 pour la défaillance totale de l'alimentation en eau des générateurs de vapeur (alimentation normale et alimentation de secours) ;

- la procédure H3 pour la défaillance totale des sources électriques externes et internes (défaillance des deux sources externes du réseau, échec de l'ilotage et défaillance des deux groupes électrogènes) ;

- la procédure H4 pour la défaillance totale du système d'injection de sécurité ou du système d'aspersion dans l'enceinte pendant la phase à long terme suivant un accident de perte de réfrigérant primaire (perte totale à terme des moyens de pompage ou d'échange de chaleur) ;

- la procédure H5 pour la protection de certains sites fluviaux à l'égard d'une crue supérieure à la crue millénale.

Au-delà des accidents traités dans le cadre indiqué ci-dessus, il subsiste des possibilités d'enchaînements d'événements pouvant conduire à des rejets radioactifs importants à l'extérieur de l'installation. C'est le cas des accidents de fusion du cœur. Des procédures traitant de situations dites « ultimes » ont été créées dans l'objectif de limiter ou de retarder l'endommagement du cœur et les conséquences radiologiques. Ces procédures sont :

- la procédure U1 pour éviter la fusion du cœur dans les situations où aucune procédure A ou $\mathrm{H}$ ne serait adaptée ou efficace. Elle préconise, en fonction de l'évolution des températures à la sortie du cœur du réacteur, et de la disponibilité des systèmes et matériels, les meilleures actions en termes d'utilisation des générateurs de vapeur, de l'injection de sécurité, des soupapes de décharge du pressuriseur et des pompes primaires pour prévenir la fusion du cœur ;

- la procédure U2 pour localiser et isoler les fuites de l'enceinte de confinement ;

- la procédure U3 pour mettre en place des moyens mobiles de secours du système d'injection de sécurité et du système d'aspersion dans l'enceinte ; elle vient en prolongement de la procédure $\mathrm{H} 4$;

- la procédure U4 pour mettre en place des dispositions pour prévenir les rejets atmosphériques précoces en cas de percée de la cuve et attaque du radier par le corium ; 
- la procédure U5 pour permettre la décompression de l'enceinte de confinement à travers le filtre à sable.

Dans une telle situation, les équipes techniques de crise utiliseraient le guide d'action des équipes de crise (GAEC) et le guide d'intervention en accident grave (GIAG) qui définit les actions spécifiques à entreprendre pour assurer, le plus longtemps possible, le confinement des produits radioactifs.

\subsubsection{Choix de la procédure et approche par état}

Pour déterminer la procédure de conduite appropriée, il est nécessaire de faire un diagnostic de l'état du réacteur, fondé sur l'analyse des alarmes et des grandeurs physiques utiles à ce diagnostic.

Pour les situations avec cumul de défaillances, le diagnostic et le choix de la bonne procédure ne sont pas toujours faciles à établir. En effet, les combinaisons d'événements résultant de défaillances multiples peuvent être reproduites à l'infini. En revanche, les états physiques possibles du réacteur sont en nombre limité. Ils peuvent être identifiés à partir d'un nombre limité de données caractérisant l'état physique des principaux composants du réacteur : sous-criticité du cœur du réacteur, inventaire d'eau dans le circuit primaire, efficacité de l'évacuation de la puissance (résiduelle), intégrité des générateurs de vapeur et inventaire d'eau dans ces derniers et étanchéité de l'enceinte de confinement. Par ailleurs, les actions à mener peuvent, en général, être déduites de la connaissance de l'état physique du réacteur, sans nécessairement avoir identifié l'enchaînement des événements y ayant conduit. L'ensemble de la démarche comprenant l'identification de l'état physique de l'installation, la définition de l'objectif de conduite en fonction de cet état et la définition des priorités en termes d'actions pour maîtriser la situation, constitue « l'approche par états ».

Dans l'approche par états, l'objectif et la stratégie de conduite peuvent être redéfinis à chaque instant, en fonction de l'évolution de la situation (état physique du réacteur, défaillances matérielles, erreurs humaines). Contrairement à l'approche par événement, la conduite n'est plus définie uniquement à partir d'un diagnostic initial portant sur la cause de l'incident ou de l'accident. Cette conduite permet de couvrir tous les incidents ou accidents thermohydrauliques (brèches dans le circuit primaire, brèches dans les circuits secondaires, échauffement du cœur, etc.), simples ou multiples, cumulés ou non avec des défaillances de systèmes, de sources électriques, ou encore des défaillances humaines.

\subsubsection{Conduite incidentelle et accidentelle}

Les procédures de conduite décrivent les opérations à mener pour rejoindre un état sûr et concernent principalement :

- la maîtrise de la réactivité par la conduite des systèmes permettant un appoint de bore dans le circuit primaire après la chute des barres ; 
- le maintien de l'inventaire d'eau dans le circuit primaire par la conduite du système de contrôle chimique et volumétrique et du système d'injection de sécurité ;

- l'évacuation de la puissance résiduelle par la conduite des systèmes de refroidissement du cœur :

- les générateurs de vapeur lorsqu'ils sont disponibles : l'énergie dégagée dans le réacteur est alors évacuée par la vaporisation de l'eau des circuits secondaires qui est ensuite refroidie par le condenseur ou rejetée dans l'atmosphère; l'appoint d'eau nécessaire est effectué soit par le système normal d'alimentation en eau (ARE), soit par le système de secours (ASG) ;

- les systèmes de sauvegarde (aspersion dans l'enceinte et injection de sécurité) en cas de perte de réfrigérant primaire ou d'indisponibilité totale des générateurs de vapeur;

- le système de refroidissement du réacteur à l'arrêt après l'arrêt du réacteur (RRA pour les tranches en exploitation - hors EPR - ou RIS BP pour EPR) ;

- le confinement des produits radioactifs par la fermeture, le cas échéant, de dispositifs contribuant à l'étanchéité de l'enceinte de confinement.

La surveillance des systèmes utilisés permet aux opérateurs de détecter les éventuelles défaillances de ceux-ci et de mettre en œuvre, si cela est possible, des moyens palliatifs pendant la réparation des systèmes défaillants.

Afin d'assurer une redondance humaine, le chef d'exploitation puis l'ingénieur de sûreté de l'installation sont appelés en salle de commande dès l'entrée dans une procédure accidentelle afin d'exercer une surveillance globale de l'évolution de la situation avec les objectifs suivants :

- vérifier que les actions nécessaires pour la sûreté sont bien effectuées ;

- s'assurer que les opérateurs appliquent bien la procédure adaptée à l'état du réacteur ;

- surveiller l'état et la disponibilité des systèmes de sûreté utilisés.

En cas d'accident présentant un risque de rejet de produits radioactifs, les équipes de crise locale et nationales sont gréées en quelques heures; elles disposent du guide d'actions des équipes de crise (GAEC) et du guide d'intervention en accident grave (GIAG) (§ 2.5.2.1).

\subsection{Conclusion}

Les paragraphes précédents présentent les composants, systèmes et circuits principaux des réacteurs à eau sous pression ainsi que leurs principes généraux de fonctionnement en situation normale et en situation accidentelle. Les réacteurs à eau sous pression sont des installations complexes, qui présentent des risques spécifiques liés à la présence de quantités importantes de produits radioactifs. À chaque étape de leur 
vie (conception, construction, exploitation, démantèlement), la sûreté doit être une préoccupation permanente pour réduire les risques, en particulier de dissémination de produits radioactifs.

À tous les stades de la vie d'un réacteur, des dispositions sont prises en vue de protéger l'homme et son environnement contre les effets de la radioactivité ; elles visent à :

- assurer le fonctionnement normal des installations ;

- prévenir les incidents et les accidents ;

- limiter les conséquences d'un incident ou accident éventuel.

L'approche utilisée pour la mise en œuvre de ces dispositions de sûreté est décrite dans le chapitre qui suit.

\section{Références}

[1] P. Coppolani, N. Hassenboehler, J. Joseph, J.-F. Petretot, J.-P. Py, J.-S. Zampa, La chaudière des réacteurs à eau sous pression, EDP Sciences, collection INSTN, 2004.

[2] J. Bourgeois, P. Tanguy, F. Cogné, J. Petit, La sûreté nucléaire en France et dans le Monde, Polytechnica, 1996.

[3] J. Libmann, Éléments de sûreté nucléaire, EDP Sciences, collection IPSN, 2000. 